This item was submitted to Loughborough's Research Repository by the author.

Items in Figshare are protected by copyright, with all rights reserved, unless otherwise indicated.

\title{
Refraction of dispersive shock waves
}

PLEASE CITE THE PUBLISHED VERSION

http://dx.doi.org/10.1016/j.physd.2012.06.002

\section{PUBLISHER}

(c) Elsevier

VERSION

AM (Accepted Manuscript)

\section{PUBLISHER STATEMENT}

This work is made available according to the conditions of the Creative Commons Attribution-NonCommercialNoDerivatives 4.0 International (CC BY-NC-ND 4.0) licence. Full details of this licence are available at: https://creativecommons.org/licenses/by-nc-nd/4.0/

\section{LICENCE}

CC BY-NC-ND 4.0

\section{REPOSITORY RECORD}

El, G.A., V.V. Khodorovskii, and Antin M. Leszczyszyn. 2019. "Refraction of Dispersive Shock Waves". figshare. https://hdl.handle.net/2134/16946. 


\title{
Refraction of dispersive shock waves
}

\author{
G.A. El, V.V. Khodorovskii and A.M. Leszczyszyn \\ Department of Mathematical Sciences, Loughborough University, \\ Loughborough, LE11 3TU, UK
}

\begin{abstract}
We study a dispersive counterpart of the classical gas dynamics problem of the interaction of a shock wave with a counter-propagating simple rarefaction wave often referred to as the shock wave refraction. The problem is considered in the framework of the one-dimensional defocusing nonlinear Schrödinger (NLS) equation and is fundamental to the understanding of 'dispersivehydrodynamic' flow interactions in superfluids and nonlinear optical media. We consider both cubic (Kerr) nonlinearity and saturable nonlinearity typical for photorefractive optical materials. For the integrable Kerr nonlinearity case we present a full asymptotic description of the dispersive shock wave (DSW) refraction by constructing appropriate exact solutions of the modulation Whitham equations. In particular, we derive a compact explicit formula for the DSW refraction phase shift, a certain analog of the soliton phase-shift in elastic two-soliton collisions. For the NLS equation with saturable nonlinearity we take advantage of the recently developed method for the dispersive shock wave description in non-integrable dispersive systems to obtain key parameters of the DSW refraction. Our modulation theory analytical results are supported by direct numerical simulations of the corresponding full dispersive initial-value problem.
\end{abstract}

\section{Introduction}

Recent developments of experimental techniques of cold-atom and laser physics and observations of a number of superfluid and optical counterparts of classical hydrodynamic phenomena such as solitons, shock waves, rarefaction waves, vortex streets etc. (see e.g. [1, 2, 3, 4, 5, 6, 7]) stimulated the growing interest in the mathematical methods and results of dispersive hydrodynamics - the theory of multiscale nonlinear flows in media with dispersive (rather than dissipative) mechanisms of regularization of breaking singularities. Central to dispersive hydrodynamics is the theory of dispersive shock waves (DSWs) (see [8] and references therein) which represent conservative oscillatory counterparts of classical (viscous) shock in fluids and gases [9], [10]. These DSWs have revealed novel dynamics and interesting interaction behaviour and have recently become an object of intensive theoretical and experimental investigations, most notably in Bose-Einstein condensates (BECs) $[1,2,11]$, where these waves represent a striking manifestation of quantum statistics on a macroscopic scale.

While the dynamics of DSWs per se have been studied in numerous works since the pioneering paper [12] by Gurevich and Pitaevskii, their interaction behaviour has begun to be investigated theoretically relatively recently. One of the first analytical results in this direction was obtained in [13], where certain cases of the 'overtaking' interaction of DSWs and rarefaction waves (RWs) have been considered in the framework of the Korteweg - de Vries (KdV) equation using appropriate exact solutions of the associated nonlinear modulation (Whitham) system. In the recent paper [14] the full classification of such unidirectional interactions of DSWs and RWs in weakly dispersive flows was made using the analytical inverse scattering transform (IST) solutions for the KdV equation and numerical solutions of the KdV-Whitham equations. This classification has revealed certain 
similarities as well as fundamental differences between classical and dispersive-hydrodynamic overtaking shock wave-rarefaction wave interactions. In many physical settings, however, one has to deal with bi-directional (head-on) wave collisions which cannot be captured by the KdV type models and should be studied in the framework of two-wave models. Such a bi-directional interaction problem represents a dispersive counterpart of the classical gas dynamics problem which is often referred to as the 'shock wave refraction'.

When a one-dimensional viscous shock wave (SW) undergoes a head-on collision with a rarefaction wave, the parameters of the two waves alter so that the long-time output of such an interaction consists of a new pair of SW and RW propagating in opposite directions. Since the SW speed changes from one constant value to another as a result of its propagation through the finite RW region with varying density and velocity, the interaction diagram in the $(x t)$-plane could be naturally interpreted as the SW refraction on the RW. As a matter of fact, the SW refraction can be observed in two-dimensional stationary flows where the effect acquires its direct geometrical significance. Refraction of SW's has been studied in many classical gas and fluid dynamics works (see, e.g. some of the original papers $[15,16,17,18,19]$ and monographs [9], [20], [21]). It must be said, however, that, while the qualitative features of the SW refraction process are well understood, its analytic description is seriously hindered due to the presence of the varying entropy region between the refracted SW and RW. As a result, the system of equations describing the head-on SW-RW interaction turns out to be so complicated that numerical solution becomes in most cases the only available resort.

In dispersive compressible dissipationless flows the entropy does not change and, in contrast to viscous gas dynamics, the bidirectional DSW-RW interaction can be described analytically in terms of solutions of the Whitham modulation equations [22] associated with the original dispersivehydrodynamic system and governing slow variations of the wave parameters (amplitude, wavenumber, mean etc.) on the scale much larger than the medium typical coherence length.

In this paper, we perform an analytical study of the head-on DSW-RW interaction in the framework of the nonlinear Schrödinger equation with defocussing, which is a fundamental mathematical model in nonlinear optics and condensed matter physics (see e.g. [23], [24]). Apart from the obvious significance as a dispersive counterpart of a classical gas dynamics problem, the theory of the DSW refraction in nonlinear Schrödinger flows could find applications in superfluid dynamics and laser optics. For the case of cubic nonlinearity the NLS equation is a completely integrable system and a full asymptotic description of the DSW-RW interaction becomes possible owing to the availability of exact solutions of the NLS-Whitham equations describing slow variations of the rapidly oscillating wave field in the interaction zone. The key element of the construction of the exact analytic solution is the mapping of the two-component reduction of the NLS-Whitham system to the classical linear Euler-Poisson-Darboux (EPD) equation. This mapping was introduced for the KdV-Whitham system in [27] [28], [29] and [30]; and for the NLS equation in [29]. It is worth noting that the same EPD equation also describes, in the hodograph plane, the interaction of two nonlinear simple waves in ideal shallow-water dynamics - see, e.g. [22].

Along with the study of the DSW refraction in Kerr media described by the integrable NLS equation, we also undertake a similar investigation of the DSW-RW interaction in the framework of the NLS equation with saturable nonlinearity (sNLS), which represents a standard model for the optical beam propagation in photorefractive crystals (see, e.g. [25], [26], [23]). The photorefractive systems have been recently used for the modelling dispersive-hydrodynamic flows in BECs by means of an all-optical setting [3] so the quantification of the contribution of the saturation effects to the 'superfluid' dynamics of light is important for the comparison with BEC experiments.

Since the sNLS equation is not integrable by the inverse scattering transform, it cannot be reduced, in the semi-classical Whitham limit, to the EPD equation. Therefore, the analytic method employed for the description of the DSW refraction in the cubic nonlinearity case is not applicable 
to the sNLS equation. To tackle the sNLS refraction problem analytically, we take advantage of the proposed in [31] approach to the dispersive Riemann problem treatment in non-integrable conservative systems. This has enabled us to derive key parameters of the refracted DSW as well as the DSW refraction acceleration and amplification coefficients as functions of the initial data and the saturation parameter $\gamma$. We note that the theory of propagation of simple photorefractive DSWs was developed in paper [32], which contains some detailed explanations of the application of the method of [31] to the sNLS equation. In the present paper, we extend the results of [32] to describe the photorefractive DSW-RW interaction. In particular, we show that for a broad range of parameters the photorefractive DSW-RW interaction is asymptotically "clean" , i.e. is not accompanied by the generation of new DSWs or RWs. Our analytical (modulation theory) results are shown to be in a good agreement with the results of direct (dispersive) numerical simulations.

\section{DSW refraction in Kerr media: formulation of the problem}

We first formulate the problem for the defocusing NLS equation with cubic (Kerr) nonlinearity

$$
i \epsilon \psi_{t}+\frac{\epsilon^{2}}{2} \psi_{x x}-|\psi|^{2} \psi=0
$$

where $\psi$ is a complex valued function and $\epsilon$ is a dimensionless dispersion parameter (coherence length). Using the Madelung transformation $\psi \mapsto(n, u)$

$$
\psi(x, t)=\sqrt{n(x, t)} \exp \left(\frac{i}{\epsilon} \int^{x} u\left(x^{\prime}, t\right) d x^{\prime}\right),
$$

where $n(x, t)>0$ and $u(x, t)$ are real-valued functions, we represent the NLS equation (1) in the "dispersive-hydrodynamic" form

$$
\begin{array}{r}
n_{t}+(n u)_{x}=0 \\
u_{t}+u u_{x}+n_{x}+\epsilon^{2}\left(\frac{n_{x}^{2}}{8 n^{2}}-\frac{n_{x x}}{4 n}\right)_{x}=0
\end{array}
$$

with the 'fluid' density $n$ and velocity $u$.

The dispersionless (classical) limit of system (3) is obtained by setting $\epsilon=0$ and is nothing but the system of ideal shallow-water equations

$$
n_{t}+(n u)_{x}=0, \quad u_{t}+u u_{x}+n_{x}=0,
$$

which can be represented in the diagonal form

$$
\frac{\partial \lambda_{ \pm}}{\partial t}+V_{ \pm}\left(\lambda_{+}, \lambda_{-}\right) \frac{\partial \lambda_{ \pm}}{\partial x}=0
$$

with the Riemann invariants

$$
\lambda_{ \pm}=\frac{1}{2} u \pm \sqrt{n}
$$

and the characteristic velocities

$$
V_{+}=\frac{3}{2} \lambda_{+}+\frac{1}{2} \lambda_{-}, \quad V_{-}=\frac{3}{2} \lambda_{-}+\frac{1}{2} \lambda_{+} .
$$

To study the bidirectional (head-on) interaction of a DSW and a rarefaction wave (RW) we consider the following configuration. Let at some moment of time say $t=t_{c}$, a simple rightpropagating DSW confined to the expanding region $x_{1}^{-}<x<x_{1}^{+}$and a simple left-propagating 


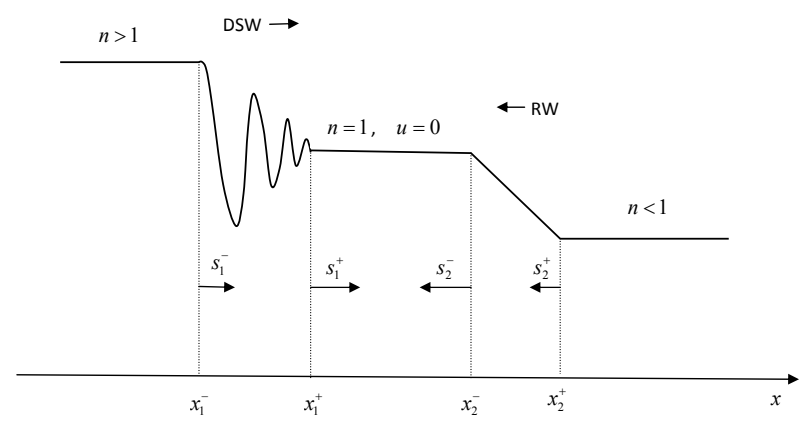

Figure 1: Sketch of the density profile in the NLS flow prior to head-on DSW-RW interaction

RW located at $x_{2}^{-}<x<x_{2}^{+}$, be separated by an undisturbed flow region $x_{1}^{+}<x<x_{2}^{-}$with $n=1$ and $u=0$ (see Fig. 1). Without much loss of generality one can assume that the DSW and RW are both centred in the $(x, t)$-plane at $(0,0)$ and $(0, l)$ respectively, so that $x_{1}^{ \pm}=s_{1}^{ \pm} t, x_{2}^{ \pm}=l+s_{2}^{ \pm} t$, where $s_{1}^{+}>s_{1}^{-}>0, s_{2}^{-}<s_{2}^{+}<0$ are the speeds of the respective DSW and RW edges. We also assume that $l \gg 1$.

The following transition conditions must be satisfied across the DSW and RW respectively (see [33], [34]):

$$
\begin{aligned}
& \lambda_{-}\left(x_{1}^{-}, t_{c}\right)=\lambda_{-}\left(x_{1}^{+}, t_{c}\right)=-1 \quad \text { simple right-propagating DSW transition } \\
& \lambda_{+}\left(x_{2}^{-}, t_{c}\right)=\lambda_{-}\left(x_{2}^{+}, t_{c}\right)=1 \quad \text { simple left-propagating RW transition }
\end{aligned}
$$

The transition conditions (8), (9) imply that the described above flow configuration can be realised as a result of the evolution of the initial flow profile $n(x, 0), u(x, 0)$ specified in terms of the shallowwater Riemann invariants $\lambda_{ \pm}$(6) having the jumps of different polarity shifted with respect one another by the distance $l$ (see Fig 2a):
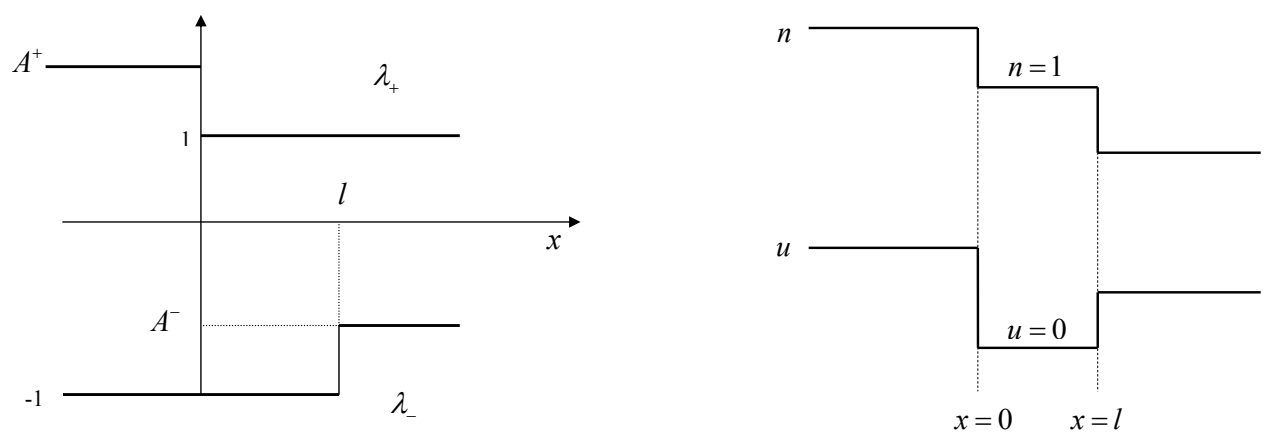

Figure 2: Initial conditions for the NLS equation (3) leading to the head-on DSW-RW interaction. Left: hydrodynamic Riemann invariants $\lambda_{ \pm}(10)$; Right: corresponding density $n$ and velocity $u$ distributions (11).

$$
\lambda_{+}(x, 0)=\left\{\begin{array}{ll}
A^{+} & \text {for } \quad x<0, \\
1 & \text { for } \quad x>0 ;
\end{array} \quad \lambda_{-}(x, 0)= \begin{cases}-1 & \text { for } \quad x<l, \\
A^{-} & \text {for } x>l,\end{cases}\right.
$$

where $A^{+}>1$ and $-1<A^{-}<1$. 
The initial conditions for $n$ and $u$ corresponding to (10) are then readily found using (6) in the form of piecewise constant distributions (see fig. $2 \mathrm{~b}$ )

$n(x, 0)=\left\{\begin{array}{ll}\frac{1}{4}\left(1+A^{+}\right)^{2}>1 & \text { for } x<0, \\ 1 & \text { for } 0<x<l \\ \frac{1}{4}\left(1-A^{-}\right)^{2}<1 & \text { for } x>l\end{array} \quad u(x, 0)= \begin{cases}A^{+}-1>0 & \text { for } x<0, \\ 0 & \text { for } 0<x<l, \\ 1+A^{-}>0 & \text { for } x>l .\end{cases}\right.$

The evolution (3), (11) can be qualitatively understood using the results of papers [33] and [34] where the Riemann problem (which is a particular case of the problem (3), (10) with $l=0$ ) was considered and a full classification for the different cases of the decay was constructed using similarity solutions of the modulation NLS-Whitham equations in the framework of "matched regularisation" procedure of the Gurevich-Pitaevskii type (see also [35], [36] for the further detailed analysis using the alternative "global regularisation" formulation). The crucial difference between the dispersive Riemann problem of [33], [34] and the problem (3), (11) considered in the present paper is that the two discontinuities for $\lambda_{+}$and $\lambda_{-}$are now spaced a large distance $l$ so the modulation problem is no longer self-similar and a more general consideration is required.

The asymptotic solution of the NLS dispersive Riemann problem obtained in [33], [34] (see also [35], [36]) and the direct numerical simulations of the more general general initial-value problem (3), (11) imply that the evolution (3), (11) will initially lead to the formation of a right-propagating simple DSW and a left-propagating simple RW as in Figs. 1,2. Both waves expand with time and start to overlap and interact at some $t=t_{0}$. The interaction continues until some $t=t^{*}>t_{0}$ when the two waves fully separate so that at $t>t^{*}$ there is a combination of new, "refracted", simple DSW and RW separated by a new constant state $n_{0} \neq 1, u_{0} \neq 0$. All the described stages of the DSW refraction are clearly seen on the direct numerical simulation plots in Fig. 3 - 5. The most obvious effect of the head-on DSW-RW interaction is the change of the key parameters (intensities, speeds) of the interacting waves. Another, more subtle, effect is the change of the modulation "phase" distributions acquired by the DSW and RW during the interaction. In particular if the incident DSW (RW) was centred at $t=0$, the refracted DSW (RW) is generally no longer a centred wave.

We also note that the outlined interaction configuration can also be realised in the framework of the dispersive piston problem (see [37], [38], [39]) involving two pistons, the right piston being pulled out from the gas with constant velocity producing thus a left-propagating rarefaction wave while the left piston being pushed into the gas producing the right propagating DSW. Another pertinent problem is the interaction of stationary two-dimensional DSW and RW forming in hypersonic dispersive flows past extended obstacles. This latter configuration is relevant to the BEC experiments [1] and can also be reformulated in terms of the already mentioned dispersive piston problem (see [40], [38]).

Our concern in the first part of the paper will be to obtain analytical description of the head-on DSW-RW interaction in terms of the initial profile parameters $A^{+}, A^{-}$and $l$.

\section{Refraction of shock waves in classical gas dynamics}

Before we proceed with the analysis of the bidirectional dispersive refraction problem (3), (11) we outline some classical results on the head-on interaction of viscous shocks and rarefaction waves (see e.g. [15], [16], [20], [21]).

Consider a one-dimensional motion of a polytropic isentropic gas, i.e. a gas with the equation of state $p=c n^{\gamma}$, where $p$ and $n$ are the gas pressure and density respectively, $\gamma$ is the adiabatic exponent and $c$ is a constant (the dispersionless shallow-water dynamics (4) is equivalent to the dynamics of the polytropic gas with $\gamma=2$ ). We consider the following flow configuration (see Fig. 

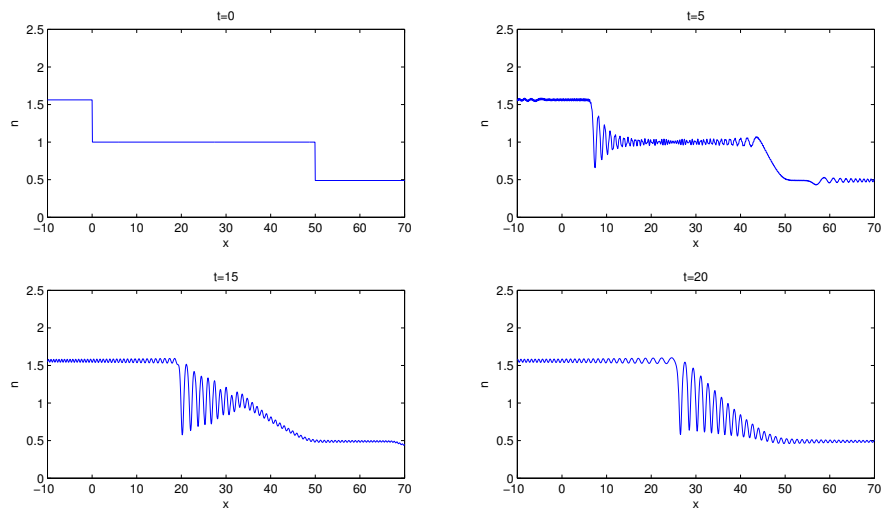

$t=35$
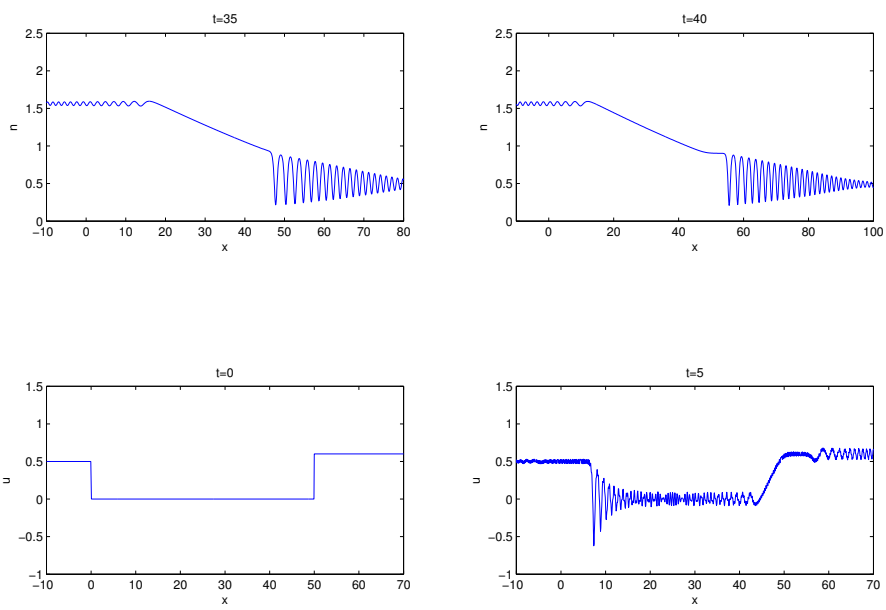

$t=15$

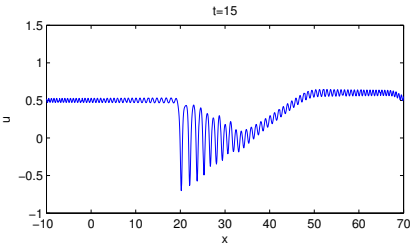

$t=35$
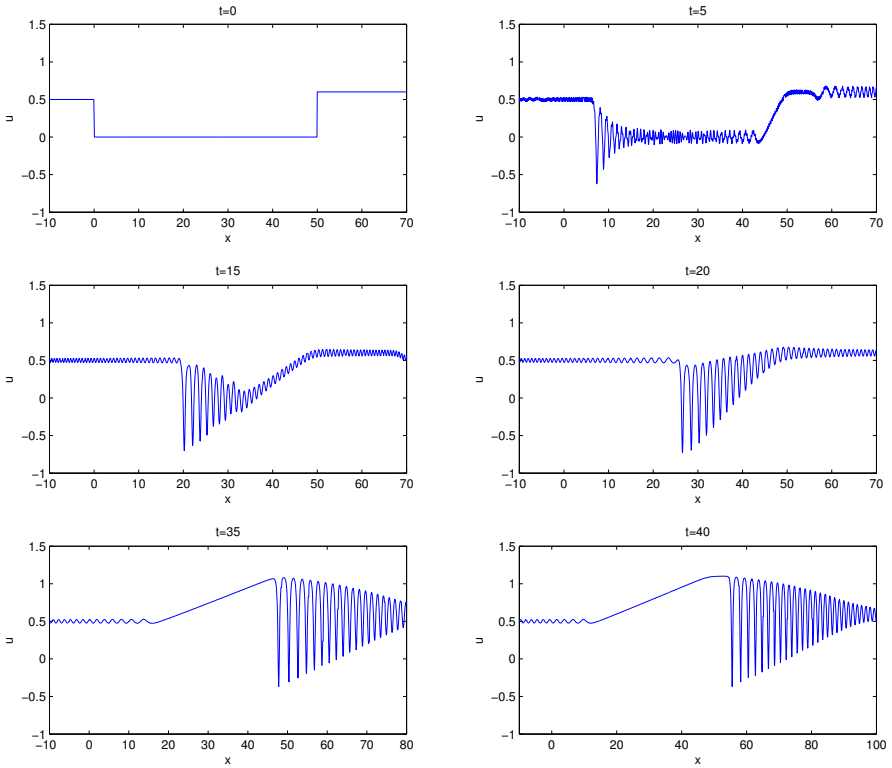

$t=20$

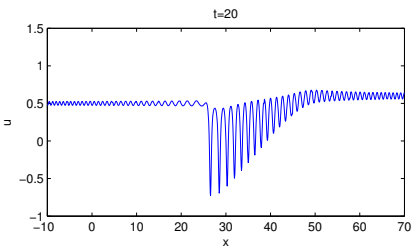

$\mathrm{t}=40$

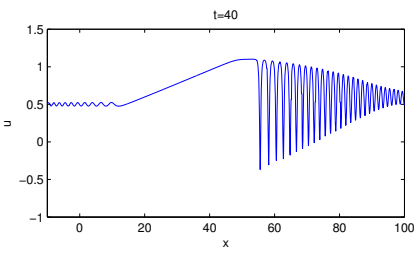

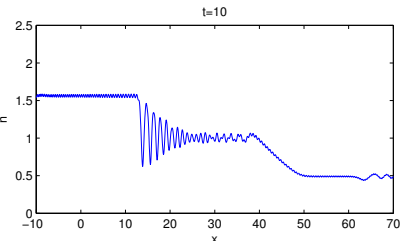

$t=25$
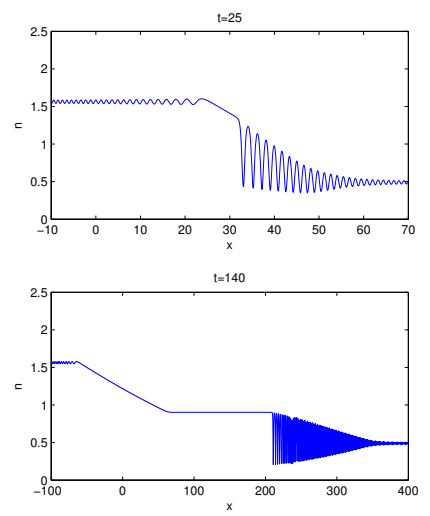

$t=10$

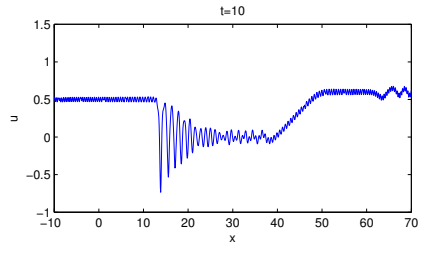

$t=25$
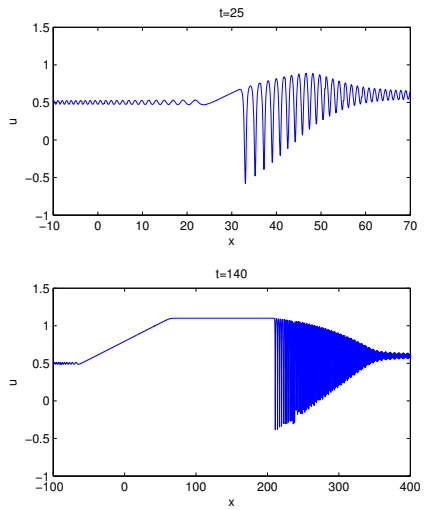

Figure 3: Bidirectional interaction of a DSW and RW: density (upper) and velocity (lower) profile; Initial data parameters: $A^{+}=1.5, A^{-}=-0.4, l=50$. The value of the dispersion parameter $\epsilon$ used in the simulations is 0.4 


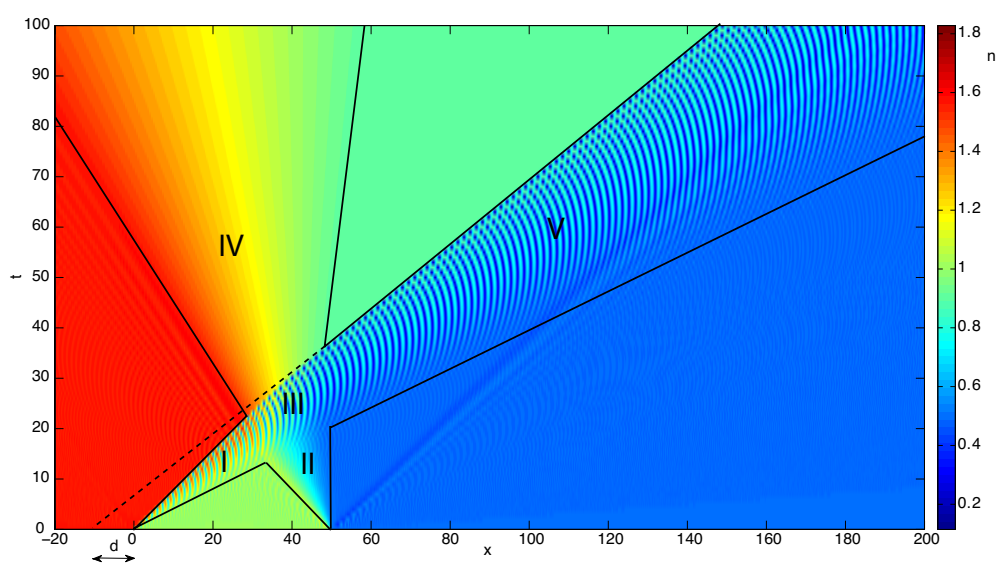

Figure 4: (Colour online) Density plot corresponding to the DSW-RW interaction shown in Fig. 3.

4). Let the gas motion at some moment of time, say $t=t_{c} \geq 0$ consist of three regions of constant flow separated by two waves: a right-propagating shock wave (SW) located at some $x=x_{c}$ and a left-propagating RW centred at $x=l$ and occupying a finite region of space (as already was mentioned, such a configuration can be created by piston motion inside a tube - see e.g. [16]). Let the density and velocity of the flow be $\left(n_{1}, u_{1}\right)$ as $x \rightarrow-\infty$ and $\left(n_{2}, u_{2}\right)$ as $x \rightarrow+\infty$. Then the gas motion at $t>t_{c}$ can be qualitatively described as follows:

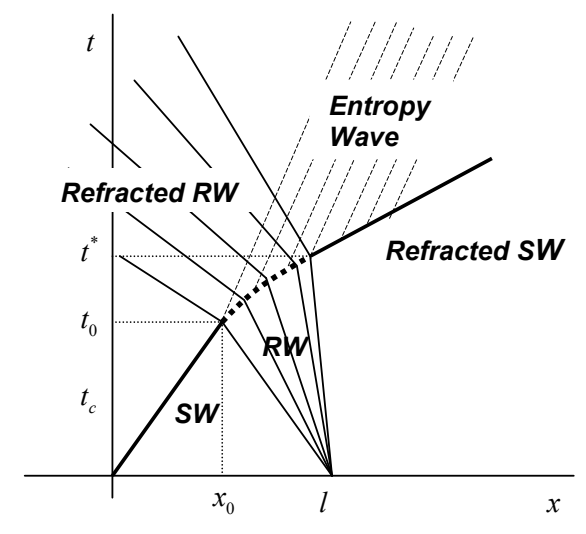

Figure 5: Head-on interaction of SW and RW in classical gas dynamics

- The SW and RW propagate independently until the moment $t=t_{0}$, when the shock enters the rarefaction wave region at some $x=x_{0}$ say. Before that moment, i.e. for $0<t<t_{0}$, the entropy undergoes a rapid constant change across the SW so the SW speed and strength (the pressure excess across it) are determined by the standard Rankine-Hugoniot conditions. The rarefaction wave is described by the centred left-propagating simple-wave solution of the inviscid hydrodynamic equations of motion. The parameters of the constant flow between the SW and RW are found at the intersection of the $n-u$ diagrams for the SW and RW (see e.g. [9]).

- During certain time interval $t_{0}<t<t^{*}$ the SW and the RW interact. The interaction 
is accompanied by the variations of the shock strength and results in the formation of the varying entropy region (the so-called 'entropy wave') behind the SW. Therefore, the flow behind the refracted SW is not isentropic.

- At $t=t^{*}$ the SW exits the RW region and the two waves again propagate separately in opposite directions, each having an altered (as compared with the values before the interaction) set of parameters. An important general result is that the speeds of the refracted SW and RW and the density/velocity jumps across them are exactly as they would have been in the corresponding origin-centred Riemann problem (i.e. in the decay of an initial discontinuity problem with the gas parameters $\left(n_{1}, u_{1}\right)$ at $x<0$ and $\left(n_{2}, u_{2}\right)$ at $\left.x>0\right)$, however, the spatial locations of the refracted waves differ from those in the corresponding Riemann problem. The refracted SW always has greater speed and strength than the original one.

As already was mentioned, the presence of the 'entropy wave' behind the refracted SW radically complicates quantitative analysis of the motion and, as a result, the SW-RW head-on collision problem can generally be treated only numerically. In contrast to classical gas dynamics, dispersive hydrodynamic flows governed by completely integrable equations often admit full analytical description. In particular, such a description is available for the DSW refraction process. This description can also be generalised (to some extent) to certain types of non-integrable dispersive equations.

\section{Single-phase modulation theory for the defocusing cubic NLS equation: account of results}

It is known very well that analytical theory of one-dimensional dispersive compressible flows containing DSWs can be constructed in the framework of the Whitham modulation equations [22]. In this section we make a brief account of the relevant results of the modulation theory for the defocusing cubic NLS equation, which will be necessary for the analysis of the DSW - RW interaction in the subsequent sections. The single-phase NLS modulation system was derived in [48], [49] (see also [35]) using the finite-gap integration methods. A more elementary derivation of this system using a reduced version of the single-gap integration can be found in [47]. Importantly, the theory developed in this section makes substantial use of the integrability of the NLS-Whitham modulation system, which is inherited from the complete integrability of the original cubic NLS equation. A different method, proposed in [31] and applicable to the description of DSWs in nonintegrable systems, will be used in Section 6 for the description of the DSW refraction in the media

described by the NLS equation with saturable nonlinearity (97), which does not enjoy the complete integrability property.

It should be noted that, since the results of the modulation theory do not depend on the value of the dispersion parameter $\epsilon$ in the NLS equation (1), we shall assume $\epsilon=1$ in the subsequent analytical representations of the periodic solutions, while in the numerical simulations we shall normally be using smaller values of $\epsilon$ to reduce the temporal scale of the DSW structure establishment.

\subsection{Periodic solution and modulation equations}

The periodic travelling wave solution of the defocusing NLS equation (3) can be expressed in terms of the Jacobi elliptic sn function and is parametrised by four integrals of motion $\lambda_{1} \leq \lambda_{2} \leq \lambda_{3} \leq \lambda_{4}$ (as was already mentioned, we assume $\epsilon=1$ in the NLS equation),

$$
n=\frac{1}{4}\left(\lambda_{4}-\lambda_{3}-\lambda_{2}+\lambda_{1}\right)^{2}+\left(\lambda_{4}-\lambda_{3}\right)\left(\lambda_{2}-\lambda_{1}\right) \operatorname{sn}^{2}\left(\sqrt{\left(\lambda_{4}-\lambda_{2}\right)\left(\lambda_{3}-\lambda_{1}\right)} \theta, m\right),
$$




$$
u=U-\frac{C}{n},
$$

where $C=\frac{1}{8}\left(-\lambda_{1}-\lambda_{2}+\lambda_{3}+\lambda_{4}\right)\left(-\lambda_{1}+\lambda_{2}-\lambda_{3}+\lambda_{4}\right)\left(\lambda_{1}-\lambda_{2}-\lambda_{3}+\lambda_{4}\right)$,

$$
\theta=x-U t-\theta_{0}, \quad U=\frac{1}{2} \sum_{i=1}^{4} \lambda_{i}
$$

$U$ being the phase velocity of the nonlinear wave and $\theta_{0}$ the initial phase.

The modulus $0 \leq m \leq 1$ of the elliptic solution (12) is defined as

$$
m=\frac{\left(\lambda_{2}-\lambda_{1}\right)\left(\lambda_{4}-\lambda_{3}\right)}{\left(\lambda_{4}-\lambda_{2}\right)\left(\lambda_{3}-\lambda_{1}\right)}
$$

and the wave amplitude is

$$
a=\left(\lambda_{4}-\lambda_{3}\right)\left(\lambda_{2}-\lambda_{1}\right) .
$$

The wavelength of the periodic wave (12) is given by

$$
\begin{gathered}
\mathfrak{L}=\int_{\lambda_{3}}^{\lambda_{4}} \frac{d \lambda}{\sqrt{\left(\lambda-\lambda_{1}\right)\left(\lambda-\lambda_{2}\right)\left(\lambda-\lambda_{3}\right)\left(\lambda_{4}-\lambda\right)}}=\int_{\lambda_{1}}^{\lambda_{2}} \frac{d \lambda}{\sqrt{\left(\lambda-\lambda_{1}\right)\left(\lambda_{2}-\lambda\right)\left(\lambda_{3}-\lambda\right)\left(\lambda_{4}-\lambda\right)}} \\
=\frac{2 \mathrm{~K}(m)}{\sqrt{\left(\lambda_{4}-\lambda_{2}\right)\left(\lambda_{3}-\lambda_{1}\right)}}
\end{gathered}
$$

$\mathrm{K}(m)$ being the complete elliptic integral of the first kind. As a matter of fact, $\mathfrak{L}>0$.

In the limit as $m \rightarrow 1$ (i.e. as $\lambda_{3} \rightarrow \lambda_{2}$ ) the travelling wave solution (12) turns into a dark soliton

$$
n=n_{s}-\frac{a_{s}}{\cosh ^{2}\left(\sqrt{a_{s}}\left(x-U_{s} t-\theta_{0}\right)\right)},
$$

where the background density $n_{s}$, the soliton amplitude $a_{s}$ and velocity $U_{s}$ are expressed in terms of $\lambda_{1}, \lambda_{2}, \lambda_{4}$ as

$$
n_{s}=\frac{1}{4}\left(\lambda_{4}-\lambda_{1}\right)^{2}, \quad a_{s}=\left(\lambda_{4}-\lambda_{2}\right)\left(\lambda_{2}-\lambda_{1}\right), \quad U_{s}=\frac{1}{2}\left(\lambda_{1}+2 \lambda_{2}+\lambda_{4}\right) .
$$

Allowing the parameters $\lambda_{1}, \lambda_{2}, \lambda_{3}, \lambda_{4}$ of the travelling wave solution (12) to be slowly varying functions of $x$ and $t$, one arrives, via the averaging or an equivalent multiple-scale perturbation procedure, at a modulated nonlinear periodic wave in which the evolution of $\boldsymbol{\lambda}=\left\{\lambda_{1}, \lambda_{2}, \lambda_{3}, \lambda_{4}\right\}$ is governed by the Whitham modulation equations $[48,49]$ (see $[22,47]$ for a detailed description of the Whitham method)

$$
\frac{\partial \lambda_{i}}{\partial t}+V_{i}(\boldsymbol{\lambda}) \frac{\partial \lambda_{i}}{\partial x}=0, \quad i=1,2,3,4
$$

$\lambda_{j}$ 's being the Riemann invariants. The characteristic velocities can be computed using the formula [29, 47]

$$
V_{i}(\boldsymbol{\lambda})=\left(1-\frac{\mathfrak{L}}{\partial_{i} \mathfrak{L}} \partial_{i}\right) U, \quad i=1,2,3,4, \quad \text { where } \quad \partial_{i} \equiv \partial / \partial \lambda_{i}
$$


Substitution of Eq. (17) into Eq. (21) yields the explicit expressions

$$
\begin{aligned}
& V_{1}=\frac{1}{2} \sum \lambda_{i}-\frac{\left(\lambda_{4}-\lambda_{1}\right)\left(\lambda_{2}-\lambda_{1}\right)}{\left(\lambda_{4}-\lambda_{1}\right)-\left(\lambda_{4}-\lambda_{2}\right) \mu(m)}, \\
& V_{2}=\frac{1}{2} \sum \lambda_{i}+\frac{\left(\lambda_{3}-\lambda_{2}\right)\left(\lambda_{2}-\lambda_{1}\right)}{\left(\lambda_{3}-\lambda_{2}\right)-\left(\lambda_{3}-\lambda_{1}\right) \mu(m)}, \\
& V_{3}=\frac{1}{2} \sum \lambda_{i}-\frac{\left(\lambda_{4}-\lambda_{3}\right)\left(\lambda_{3}-\lambda_{2}\right)}{\left(\lambda_{3}-\lambda_{2}\right)-\left(\lambda_{4}-\lambda_{2}\right) \mu(m)}, \\
& V_{4}=\frac{1}{2} \sum \lambda_{i}+\frac{\left(\lambda_{4}-\lambda_{3}\right)\left(\lambda_{4}-\lambda_{1}\right)}{\left(\lambda_{4}-\lambda_{1}\right)-\left(\lambda_{3}-\lambda_{1}\right) \mu(m)},
\end{aligned}
$$

where $\mu(m)=\mathrm{E}(m) / \mathrm{K}(m), \mathrm{E}(m)$ being the complete elliptic integral of the second kind. The characteristic velocities (22) are real for all values of the Riemann invariants, therefore system (20) is hyperbolic. Moreover, it is not difficult to show using representation (21) that

$$
\partial_{i} V_{i}>0 \text { for all } i
$$

so the NLS-Whitham system (20), (22) is genuinely nonlinear [51]. Indeed, differentiating (21) we get:

$$
\partial_{i} V_{i}=\frac{\mathfrak{L}}{2\left(\partial_{i} \mathfrak{L}\right)^{2}} \partial_{i i}^{2} \mathfrak{L} .
$$

Using the integral representations (17) for $\mathfrak{L}$ one can readily see that $\partial_{i i}^{2} \mathfrak{L}>0$ for all $i$ (it is convenient to use the first representation for the differentiations with respect $\lambda_{1}$ and $\lambda_{2}$ and the second one for the differentiations with respect $\lambda_{3}$ and $\lambda_{4}$ ), which immediately implies (23).

Also, using (21) and the intergral representations (17) one can readily show by a direct calculation that

$$
i>j \text { implies } V_{i}>V_{j} .
$$

Thus, the ordering $\lambda_{1} \leq \lambda_{2} \leq \lambda_{3} \leq \lambda_{4}$ of the Riemann invariants implies a similar ordering $V_{1} \leq V_{2} \leq V_{3} \leq V_{4}$ for the characteristic velocities. We note that the properties (23) and (25) were established in [46], [35] using the finite-gap integration framework for the derivation of the Whitham equations.

For the DSW analysis in the subsequent sections we shall need the reductions of formulae (22) for the limiting cases $m=0$ (harmonic limit) and $m=1$ (soliton limit).

The harmonic limit $m=0$ can be achieved in one of the two possible ways: either via $\lambda_{2}=\lambda_{1}$ or via $\lambda_{3}=\lambda_{4}$. Then:

$$
\begin{aligned}
& \text { when } \lambda_{2}=\lambda_{1}: \quad V_{2}=V_{1}=\lambda_{1}+\frac{\lambda_{3}+\lambda_{4}}{2}+\frac{2\left(\lambda_{3}-\lambda_{1}\right)\left(\lambda_{4}-\lambda_{1}\right)}{2 \lambda_{1}-\lambda_{3}-\lambda_{4}}, \\
& \qquad V_{3}=\frac{3}{2} \lambda_{3}+\frac{1}{2} \lambda_{4}=V_{-}\left(\lambda_{3}, \lambda_{4}\right), \quad V_{4}=\frac{3}{2} \lambda_{4}+\frac{1}{2} \lambda_{3}=V_{+}\left(\lambda_{3}, \lambda_{4}\right) . \\
& \text { when } \lambda_{3}=\lambda_{4}: \quad V_{3}=V_{4}=\lambda_{4}+\frac{\lambda_{1}+\lambda_{2}}{2}+\frac{2\left(\lambda_{4}-\lambda_{2}\right)\left(\lambda_{4}-\lambda_{1}\right)}{2 \lambda_{4}-\lambda_{2}-\lambda_{1}}, \\
& \qquad V_{1}=\frac{3}{2} \lambda_{1}+\frac{1}{2} \lambda_{2}=V_{-}\left(\lambda_{1}, \lambda_{2}\right), \quad V_{2}=\frac{3}{2} \lambda_{2}+\frac{1}{2} \lambda_{1}=V_{+}\left(\lambda_{1}, \lambda_{2}\right) .
\end{aligned}
$$


In the soliton limit we have $m=1$. This can happen only if $\lambda_{2}=\lambda_{3}$, so we obtain:

$$
\begin{array}{r}
\text { when } \lambda_{2}=\lambda_{3}: \quad V_{2}=V_{3}=\frac{1}{2}\left(\lambda_{1}+2 \lambda_{2}+\lambda_{4}\right)=U_{s}, \\
V_{1}=\frac{3}{2} \lambda_{1}+\frac{1}{2} \lambda_{4}=V_{-}\left(\lambda_{1}, \lambda_{4}\right), \quad V_{4}=\frac{3}{2} \lambda_{4}+\frac{1}{2} \lambda_{1}=V_{+}\left(\lambda_{1}, \lambda_{4}\right) .
\end{array}
$$

Thus, in both harmonic and soliton limits the fourth-order modulation system (20), (22) reduces to the system of three equations, two of which are decoupled. Moreover, one can see that in all considered limiting cases the decoupled equations agree with the dispersionless limit of the NLS equation (1). This property makes possible matching of the modulation solution with the solution to the dispersionless limit equations at the points where $m=0$ or $m=1$.

\subsection{Free-boundary matching conditions for the modulation equations}

In the description of a DSW, the Whitham equations (20) must be endowed with certain initial or boundary conditions for the Riemann invariants $\lambda_{i}$. We shall be using the Gurevich-Pitaevskii type boundary-value (matching) problem first formulated in [12] for the KdV dispersive shock waves and extended to the NLS case in [33]. A different type of the problem formulation (the so-called regularised initial-value problem for the NLS-Whitham equations) proposed in [35] and recently used in [36], [45] for the numerical analysis of the DSW interaction is less convenient for our purposes as the analytical description of the interaction zone requires the hodograph solutions of the Whitham equations, and the poor compatibility of the initial-value problems with the hodograph transform is well known (see e.g. [22]). The Gurevich-Pitaevskii matching conditions, on the contrary, are ideally compatible with the hodograph transform as they turn into the classical Goursat type characteristic boundary conditions on the hodograph plane (see [28], [29], [41], [38]). It is clear that both formulations (regularised initial-value problem for the Whitham equations and the Gurevich-Pitaevskii type matching problem) must be equivalent, although we are not aware of the rigorous proof of this equivalence.

To be specific, we shall formulate boundary (matching) conditions for the right-propagating DSW. Without loss of generality we assume that the formation of the DSW starts at the origin of the $(x, t)$-plane. In the Gurevich-Pitaevskii setting, the upper $(x, t)$-half plane is split into three regions (see Fig. 2): $\left(-\infty, x^{-}(t)\right),\left[x^{-}(t), x^{+}(t)\right]$ and $\left(x^{+}(t),+\infty\right)$.

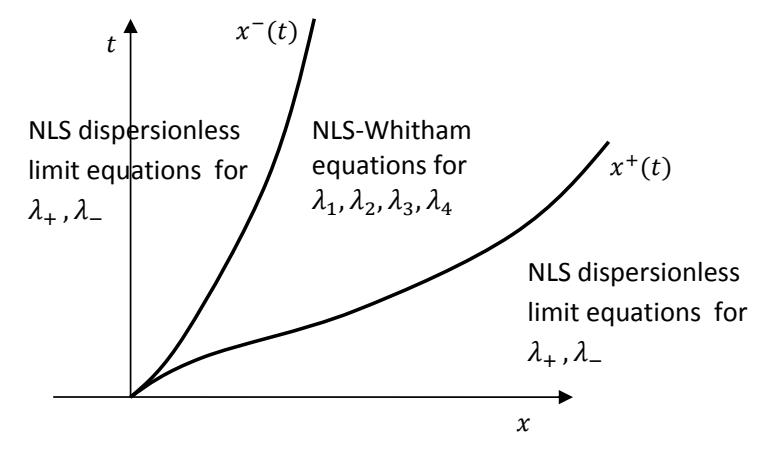

Figure 6: Splitting of the $x t$-plane in the Gurevich-Pitaevskii problem for the defocusing NLS equation.

In the "outer" regions $\left(-\infty, x^{-}(t)\right)$ and $\left(x^{+}(t),+\infty\right)$ the flow is governed by the dispersionless limit of the NLS equation, i.e. by the shallow-water system (5), (7) for the Riemann invariants 
$\lambda_{ \pm}$. In the DSW region $\left[x^{-}(t), x^{+}(t)\right]$ the averaged oscillatory flow is described by four Whitham equations (20) for the Riemann invariants $\lambda_{j}$ with the following matching conditions at the trailing $x^{-}(t)$ and leading $x^{+}(t)$ edges of the DSW (see [33, 41] for details):

$$
\begin{array}{llll}
\text { At } x=x^{-}(t): & \lambda_{3}=\lambda_{2}, & \lambda_{4}=\lambda_{+}, & \lambda_{1}=\lambda_{-}, \\
\text {At } x=x^{+}(t): & \lambda_{3}=\lambda_{4}, & \lambda_{2}=\lambda_{+}, & \lambda_{1}=\lambda_{-} .
\end{array}
$$

Here $\lambda_{ \pm}(x, t)$ are the Riemann invariants of the dispersionless limit of the NLS equation in the hydrodynamic form $(5),(7)$. The free boundaries $x^{ \pm}(t)$ of the DSW are defined by the kinematic conditions

$$
\frac{d x^{-}}{d t}=V_{2}\left(\lambda_{1}, \lambda_{2}, \lambda_{2}, \lambda_{4}\right)=V_{3}\left(\lambda_{1}, \lambda_{2}, \lambda_{2}, \lambda_{4}\right), \quad \frac{d x^{+}}{d t}=V_{3}\left(\lambda_{1}, \lambda_{2}, \lambda_{4}, \lambda_{4}\right)=V_{4}\left(\lambda_{1}, \lambda_{2}, \lambda_{4}, \lambda_{4}\right)
$$

and so are multiple characteristics of the Whitham system. The multiple characteristic velocities $V_{2}=V_{3}$ and $V_{3}=V_{4}$ in (30) are explicitly given by equations (28) and (27) respectively. One should stress that determination of $x^{ \pm}(t)$ is an inherent part of the construction of the full modulation solution. We also emphasize that matching conditions (29) are consistent with the structure of the Whitham system (20), (22) in the limiting cases $m=0$ and $m=1$ (see (27), (28)) and with the spatial oscillatory profile of the DSW in the defocusing NLS dispersive hydrodynamics (as is known very well, such a DSW has a dark soliton $(m=1)$ at the trailing edge and degenerates into the vanishing amplitude harmonic wave $(m=0)$ at the leading edge - see $[33,34,44,2])$.

\subsection{Hodograph transform and mapping to the Euler-Poisson-Darboux equation}

The hydrodynamic type modulation system (20), (22) can be reduced to a system of linear partial differential equations using the (generalised) hodograph transform [43]. We first fix two of the Riemann invariants, say

$$
\lambda_{i}=\lambda_{i 0}=\text { constant }, \quad \lambda_{j}=\lambda_{j 0}=\text { constant }, \quad i \neq j,
$$

to reduce $(20)$ to the system of two equations for the remaining two invariants $\lambda_{k}(x, t)$ and $\lambda_{l}(x, t)$, $k \neq l \neq i \neq j$

$$
\frac{\partial \lambda_{k}}{\partial t}+V_{k}\left(\lambda_{k}, \lambda_{l}\right) \frac{\partial \lambda_{k}}{\partial x}=0, \quad \frac{\partial \lambda_{l}}{\partial t}+V_{l}\left(\lambda_{k}, \lambda_{l}\right) \frac{\partial \lambda_{l}}{\partial x}=0
$$

where $V_{k, l}\left(\lambda_{k}, \lambda_{l}\right) \equiv V_{k, l}\left(\lambda_{i 0}, \lambda_{j 0}, \lambda_{k}, \lambda_{l}\right)$. Applying the hodograph transform to system (32) we arrive at a linear system for $x\left(\lambda_{k}, \lambda_{l}\right), t\left(\lambda_{k}, \lambda_{l}\right)$,

$$
\frac{\partial x}{\partial \lambda_{k}}-V_{l}\left(\lambda_{k}, \lambda_{l}\right) \frac{\partial t}{\partial \lambda_{k}}=0, \quad \frac{\partial x}{\partial \lambda_{l}}-V_{k}\left(\lambda_{k}, \lambda_{l}\right) \frac{\partial t}{\partial \lambda_{l}}=0 .
$$

Note that the hodograph transform requires that $\partial_{x} \lambda_{k, l} \neq 0$. Now we make in (33) the change of variables

$$
x-V_{k} t=W_{k}, \quad x-V_{l} t=W_{l},
$$

which reduces it to a symmetric system for $W_{k}\left(\lambda_{k}, \lambda_{l}\right), W_{l}\left(\lambda_{k}, \lambda_{l}\right)$ :

$$
\frac{\partial_{k} W_{l}}{W_{k}-W_{l}}=\frac{\partial_{k} V_{l}}{V_{k}-V_{l}} ; \quad k \neq l ; \quad \partial_{k} \equiv \partial / \partial \lambda_{k}
$$

The symmetry between $V_{l}$ and $W_{l}$ in (35) and the 'potential' structure (21) of the vector function $\left(V_{k}, V_{l}\right)$ implies the possibility of introducing a single scalar function $g\left(\lambda_{k}, \lambda_{l}\right)$ instead of the vector $\left(W_{k}, W_{l}\right)$ :

$$
W_{i}=\left(1-\frac{\mathfrak{L}}{\partial_{i} \mathfrak{L}} \partial_{i}\right) g, \quad i=k, l,
$$


or, which is the same (use (21)),

$$
W_{i}=g+2\left(V_{i}-U\right) \partial_{i} g, \quad i=k, l .
$$

Then substituting (21), (36) into (35) we arrive, taking into account (17), at the Euler-PoissonDarboux (EPD) equation for $g\left(\lambda_{k}, \lambda_{l}\right)$, first derived in the present NLS context in [29] (see also [41])

$$
2\left(\lambda_{l}-\lambda_{k}\right) \partial_{k l}^{2} g=\partial_{l} g-\partial_{k} g .
$$

Note that system (32) essentially describes the interaction of two simple waves of modulation of the NLS equation so the fact that this system reduces, in the hodograph plane, to the EPD equation (38) describing interaction of two simple waves in classical dispersionless shallow-water theory (or in gas dynamics of polytropic isentropic gas with $\gamma=2$ ) (see [22] for instance) is quite remarkable.

The general solution of the EPD equation (38) can be represented in the form (see, for instance, [50])

$$
g=\int_{a_{1}}^{\lambda_{k}} \frac{\phi_{1}(\lambda) d \lambda}{\sqrt{\left(\lambda-\lambda_{k}\right)\left(\lambda_{l}-\lambda\right)}}+\int_{a_{2}}^{\lambda_{l}} \frac{\phi_{2}(\lambda) d \lambda}{\sqrt{\left(\lambda-\lambda_{k}\right)\left(\lambda_{l}-\lambda\right)}},
$$

where $\phi_{1,2}(\lambda)$ are arbitrary (generally, complex-valued) functions and $a_{1,2}$ are arbitrary constants (which could be absorbed into $\phi_{1,2}$ ).

As a matter of fact, the same construction can be realized for any pair of Riemann invariants while the two remaining invariants are fixed. Moreover, equations (34) - (35) and further (21) - (38) turn out to be valid even when all four Riemann invariants vary [29, 41]. This becomes possible for two reasons. Firstly, the NLS modulation system (20), (21) is integrable via the generalized hodograph transform [43] which reduces it to overdetermined consistent system (35), where $k, l=1,2,3,4, k \neq l$. Secondly, the "potential" structure of the characteristic speeds $(21)$ makes it possible to use the same substitution (36) for all $k=1,2,3,4$ which results in the consistent system of six EPD equations (38) involving all pairs $\lambda_{k}, \lambda_{l}, k \neq l$.

Thus, the problem of integration of the nonlinear Whitham system (20) with rather complicated coefficients (22) is essentially reduced to solving the classical linear EPD equation (38) so in practice one needs to express the functions $\phi_{1,2}(\lambda)$ in the general solution (39) in terms of the initial or boundary conditions for the NLS equation (1). As was shown in [29], [41] (see also [38]) the freeboundary nonlinear matching conditions (29) are most conveniently translated into a classical linear Goursat characteristic boundary problem for the EPD equation (38). This enables one to find the unknown functions $\phi_{1,2}(\lambda)$ in terms of Abel transforms of the initial data.

In conclusion of this section we note that hodograph solutions do not include the special family of the simple-wave solutions as the latter correspond to the vanishing Jacobian of the hodograph transform $\left(\lambda_{k}, \lambda_{l}\right) \mapsto(x, t)$ (see, for instance, [22]).

\subsection{Modulation phase shift}

In the modulated wave, the initial phase $\theta_{0}$ of the periodic solution (12) - (15) is no longer an independent constant parameter but rather a slow function of $x, t$ so it is better described as the modulation phase shift. As was shown in [57], the function $\theta_{0}(x, t)$ can be found from the requirement that the local wavenumber $k=2 \pi / \mathfrak{L}$ and the local frequency $\omega=k U$ in the modulated wave (12) must satisfy the generalised phase relationships

$$
k=\Theta_{x}, \quad \omega=-\Theta_{t},
$$

where

$$
\Theta=k \theta=k x-\omega t-k \theta_{0}
$$


is the angular phase. Relationships (40) imply the 'conservation of waves' law

$$
k_{t}+\omega_{x}=0
$$

which is consistent with the modulation system (20) and thus yields the representation $V_{i}=\partial_{i} \omega / \partial_{i} k$ for the characteristic speeds, equivalent to (21).

For the general modulation relationship (40) to be consistent with the linear $x, t$-dependence of the phase (41) entering the local single-phase NLS solutions (12), (15) one must assume $\theta_{0}(x, t)=$ $\vartheta_{0}\left(\lambda_{1}, \lambda_{2}, \lambda_{3}, \lambda_{4}\right)$, which implies that the phase shift is completely determined by the evolution of the Riemann invariants $\lambda_{j}(x, t)$ in the modulation solution. To find $\vartheta_{0}\left(\lambda_{1}, \lambda_{2}, \lambda_{3}, \lambda_{4}\right)$ we differentiate (41) with respect to $x$ to obtain

$$
\Theta_{x}=k+\sum_{i=1}^{4}\left\{x \partial_{i} k-t \partial_{i} \omega-\vartheta_{0} \partial_{i} k-k \partial_{i} \vartheta_{0}\right\} \partial_{x} \lambda_{i}
$$

Comparing (43) with (40) we obtain for any pair $i, j, i \neq j$

$$
x \partial_{i} k-t \partial_{i} \omega-\vartheta_{0} \partial_{i} k-k \partial_{i} \vartheta_{0}=0, \quad x \partial_{j} k-t \partial_{j} \omega-\vartheta_{0} \partial_{j} k-k \partial_{j} \vartheta_{0}=0,
$$

provided $\partial_{x} \lambda_{i, j} \neq 0$. On using $\partial_{i} \omega / \partial_{i} k=V_{i}$ and $k=2 \pi / \mathfrak{L}$ system (44) is readily transformed to the form

$$
x-V_{n} t=\left(1-\frac{\mathfrak{L}}{\partial_{n} \mathfrak{L}} \partial_{n}\right) \vartheta_{0}, \quad n=i, j, \quad i \neq j .
$$

Comparison of expression (45) with the modulation hodograph solution (34), (36) enables one to identify the modulation phase shift $\vartheta_{0}(\boldsymbol{\lambda})=\theta_{0}(x, t)$ with the solution $g(\boldsymbol{\lambda})$ to the relevant boundary value problem for the $\mathrm{EPD}$ equation (38), i.e.

$$
\theta_{0}(x, t)=g(\boldsymbol{\lambda}(x, t))
$$

One can also see from (45) that one should set $\theta_{0}=0$ for a simple centred DSW described by the modulation solution in which all but one Riemann invariants are constants and the varying invariant, say $\lambda_{m}$, is implicitly specified by the equation $x-V_{m} t=0$. The condition $\theta_{0}=0$ then implies that in the dispersive Riemann (decay of a step) problem the DSW trailing dark soliton (18) is centred exactly at the trailing edge $x^{-}(t)$ defined by (30), (28).

\section{Interaction of DSW and RW: modulation solution}

\subsection{Before interaction, $0<t<t_{0}$}

At $t=0$, a simple origin-centred right-propagating DSW is generated due to the jump of the Riemann invariant $\lambda_{+}$while the jump of $\lambda_{-}$produces a similarity "shallow-water" rarefaction wave centred at $x=l$ and propagating to the left.

The similarity modulation solution describing the DSW has the form [33], [34]

$$
\begin{array}{r}
\lambda_{1}=-1, \quad \lambda_{2}=1, \quad \lambda_{4}=A^{+}, \\
\frac{x}{t}=V_{3}\left(-1,1, \lambda_{3}, A^{+}\right)=\frac{\lambda_{3}+A^{+}}{2}-\frac{\left(A^{+}-\lambda_{3}\right)\left(\lambda_{3}-1\right)}{\lambda_{3}-1-\left(A^{+}-1\right) \mu(m)},
\end{array}
$$

where

$$
m=\frac{2\left(A^{+}-\lambda_{3}\right)}{\left(A^{+}-1\right)\left(\lambda_{3}+1\right)} .
$$




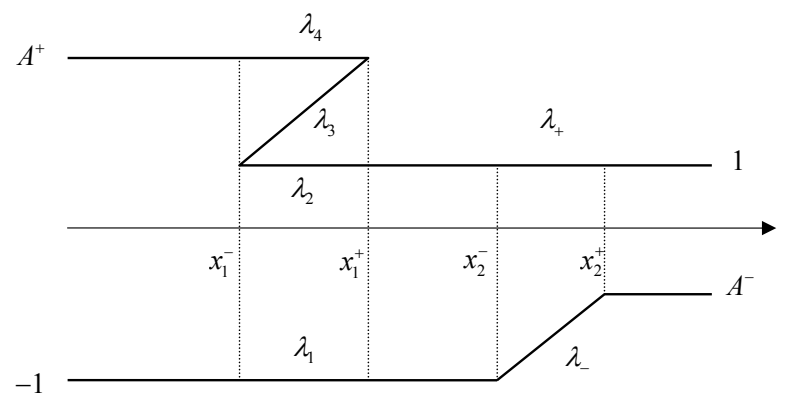

Figure 7: Schematic behaviour for the Riemann invariants before the interaction of the DSW and RW, $0<t<t_{0}$.

The boundaries of the DSW are then found from (47) by setting $\lambda_{3}=A^{+}$(i.e. $m=0$ ) for the leading edge $x_{1}^{+}$and $\lambda_{3}=1$ (i.e. $m=1$ ) for the trailing edge $x_{1}^{-}$:

$$
x_{1}^{-}=\frac{1+A^{+}}{2} t, \quad x_{1}^{+}=\left(2 A^{+}-\frac{1}{A^{+}}\right) t .
$$

The dark soliton at the trailing edge $x_{1}^{-}$of the DSW has the amplitude $a_{s}$ and rides on the background $n_{s}$ defined by (see (19))

$$
n_{s}=\left(1+A^{+}\right)^{2} / 4, \quad a_{s}=2\left(A^{+}-1\right) .
$$

The value $A^{+}=3$ corresponds to the formation of a vacuum point at the trailing edge of the DSW [34] so that the density at the dark soliton minimum is $n_{s}-a_{s}=0$. For $A^{+}>3$ the vacuum point occurs inside the DSW at some $x=x_{v}$, where $x^{-}<x_{v}<x^{+}$- see details in [34], [2].

We define the relative intensity (hereafter - simply intensity) $I$ of a DSW as the density ratio across it:

$$
I=\frac{n_{1}}{n_{2}}
$$

where $n_{1}$ and $n_{2}$ are the values of density upstream and downstream the DSW respectively. This definition can be related to the one accepted in classical gas dynamics, where the relative pressure excess across the SW is often used as a measure of the SW strength. One should, however, stress that the notion of the DSW intensity for the NLS flows retains its original meaning only for DSWs not containing vacuum points. The modification of the flow resulting from the vacuum point appearance will be discussed below in Section 5.3.3.

For the incident DSW (i.e. before the interaction) we obviously have $n_{1}=n_{s}$ and $n_{2}=1$, i.e. its intensity is

$$
I_{0}=\frac{\left(1+A^{+}\right)^{2}}{4}
$$

Now we turn to the left-propagating rarefaction wave, which is asymptotically described by the centred at $x=l$ similarity solution of the classical limit equations (5), (7) (see, e.g., [9], [10]) :

$$
\begin{array}{rlrl} 
& \lambda_{+}=1, & \\
\lambda_{-}=-1, & x<x_{2}^{-} ; \\
\frac{x-l}{t}=V_{-}\left(\lambda_{-}, 1\right)=\frac{3 \lambda_{-}+1}{2}, & x_{2}^{-} \leq x \leq x_{2}^{+} ; \\
\lambda_{-}=A^{-}, & x>x_{2}^{+} .
\end{array}
$$


Here the boundaries $x_{2}^{ \pm}$are specified by the formulae:

$$
x_{2}^{-}=l-t, \quad x_{2}^{+}=l+\frac{3 A^{-}+1}{2} t .
$$

It is instructive to note that, since the modulation system (20) in the harmonic limit is consistent with the shallow-water equations (5), (6) - see (27), the RW solution (53), (54) is also a solution of full modulation system (20), namely

$$
\lambda_{3}=\lambda_{4}=A^{+} ; \quad \lambda_{2}=\lambda_{+}=1, \quad \lambda_{1}=\lambda_{-}(x, t) .
$$

This identification of the RW solution of the dispersionless limit equations as a particular solution of the full modulation system will be used in Section 5.2.

One can readily see that $d x_{1}^{+} / d t>d x_{2}^{-} / d t$ (this also follows from the characteristic velocity ordering described in Section IV A) so the DSW will start overtaking the RW at some moment $t=t_{0}$ when the leading edge of the DSW will catch up the trailing edge of the RW at $x_{0}=x_{1}^{+}\left(t_{0}\right)=x_{2}^{-}\left(t_{0}\right)$. Using (49) and (55) we obtain

$$
t_{0}=\frac{A^{+} l}{2\left(A^{+}\right)^{2}+A^{+}-1}, \quad x_{0}=\frac{2\left(A^{+}\right)^{2}-1}{2\left(A^{+}\right)^{2}+A^{+}-1} l .
$$

\subsection{Interaction, $t_{0}<t<t^{*}$}

At $t=t_{0}$ the DSW enters the RW region so that at $t>t_{0}$ a nonlinear interaction zone confined to the interval $\left[x_{2}^{-}, x_{1}^{+}\right]$forms (see Fig. 8) and evolves until some moment $t^{*}$ when the DSW completely overtakes the RW so that $x_{2}^{+}\left(t^{*}\right)=x_{1}^{-}\left(t^{*}\right)$. At $t>t^{*}$ the DSW and RW fully separate, each acquiring a new set of parameters $\lambda_{j}$ compared to their initial characterization. One should stress that, for $t>t_{0}$ the functions $x_{1}^{ \pm}(t)$ and $x_{2}^{ \pm}(t)$ are no longer described by the formulae (49), (55) from the previous subsection.

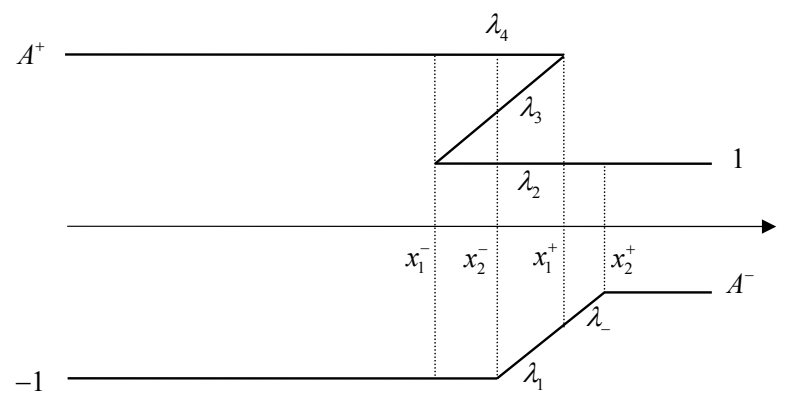

Figure 8: Schematic behaviour of the Riemann invariants during interaction of the DSW and RW, $t_{0}<t<t_{*}$.

The corresponding interaction diagram in the $x t$ plane is shown in Fig. 9 (left).

In the interaction region $\left[x_{2}^{-}, x_{1}^{+}\right]$one still has $\lambda_{2}=1$ and $\lambda_{4}=A^{+}$but the remaining two Riemann invariants $\left(\lambda_{1}\right.$ and $\left.\lambda_{3}\right)$ now vary so the modulation solution is no longer self-similar and a more general, hodograph solution (34) is needed. This is found via additional transformation (36) reducing Tsarev's equations (35) for $W_{1,3}\left(\lambda_{1}, \lambda_{3}\right) \equiv W_{1,3}\left(\lambda_{1}, 1, \lambda_{3}, A^{+}\right)$to the EPD equation (38). The general solution (39) of the EPD equation is parametrised by two arbitrary functions $\phi_{1,2}(\lambda)$ which should be found from appropriate boundary conditions. These conditions, in their turn, must 

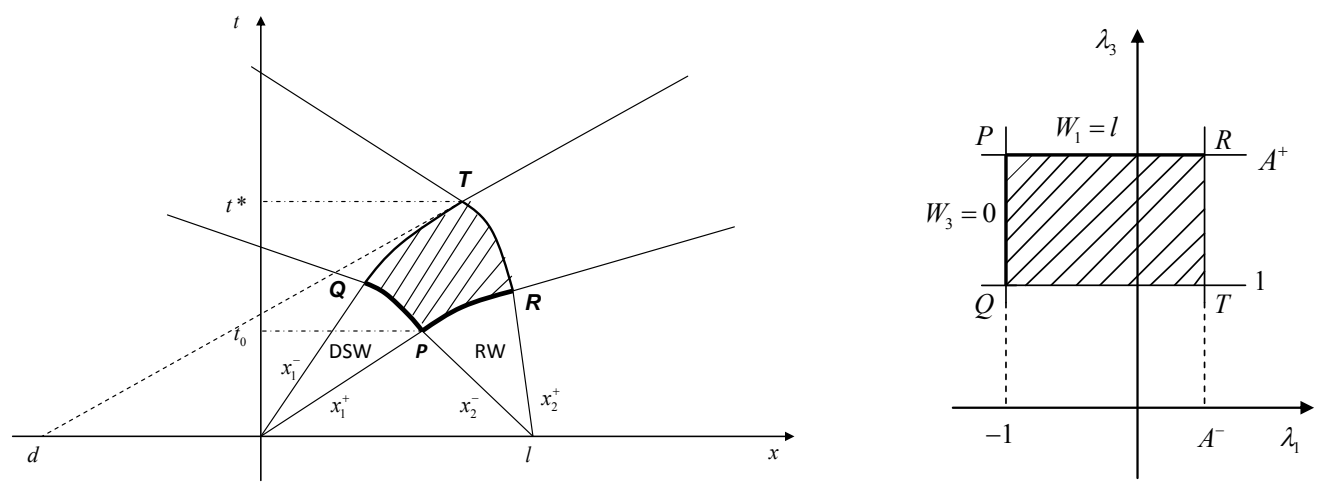

Figure 9: DSW-RW interaction diagram. Left: physical, $(x t)$ plane; Right: hodograph, $\left(\lambda_{1} \lambda_{3}\right)$ plane.

follow from the continuity matching conditions for $\lambda_{1}$ and $\lambda_{3}$ at the unknown boundaries $x_{2}^{-}(t)$ and $x_{1}^{+}(t)$.

At the left boundary $x=x_{2}^{-}(t)$ of the interaction zone (segment $P Q$ in the interaction diagram in Fig. 9, left) we have

$$
\lambda_{1}=-1, \quad \lambda_{2}=1, \quad \lambda_{3}=\lambda_{3}^{s}\left(x_{2}^{-}(t), t\right), \quad \lambda_{4}=A^{+},
$$

where $\lambda_{3}^{s}(x, t)=\lambda_{3}(x / t)$ is found from the similarity modulation solution (47). At the right boundary $x=x_{1}^{+}(t)$ of the interaction zone (segment $P R$ in Fig. 9 left) we have, similar to the second condition $(29)$,

$$
\lambda_{1}=\lambda_{-}^{r}\left(x_{1}^{+}(t), t\right), \quad \lambda_{2}=1, \quad \lambda_{3}=\lambda_{4}=A^{+},
$$

and $\lambda_{-}^{r}(x, t)=\lambda_{-}\left(\frac{x-l}{t}\right)$ is found from the rarefaction wave solution (53).

We now need to translate nonlinear free-boundary conditions (58) and (59) into the boundary conditions for the function $g\left(\lambda_{1}, \lambda_{3}\right)$ satisfying the EPD equation

$$
2\left(\lambda_{3}-\lambda_{1}\right) \partial_{13}^{2} g=\partial_{3} g-\partial_{1} g, \quad \partial_{j} \equiv \partial / \partial \lambda_{j} .
$$

This is done in two steps. First we derive the boundary conditions for the functions $W_{1}\left(\lambda_{1}, \lambda_{3}\right)$ and $W_{3}\left(\lambda_{1}, \lambda_{3}\right)$ defining the hodograph solution (34),

$$
x-V_{1} t=W_{1}, \quad x-V_{3} t=W_{3} .
$$

Using the boundary condition at $x=x_{1}^{+}$(59) and expression (27) for the characteristic velocity $V_{1}$ in the degenerate case when $\lambda_{3}=\lambda_{4}$, the first equation (61) becomes

$$
x-\frac{3 \lambda_{1}+1}{2} t=W_{1}\left(\lambda_{1}, A^{+}\right) .
$$

Since according to the matching condition (59) one has $\lambda_{1}=\lambda_{-}$at $x=x_{1}^{+}$, we get, by comparing (62) with the rarefaction wave solution (53), that

$$
W_{1}\left(\lambda_{1}, A^{+}\right)=l .
$$

Next we turn to the boundary condition (58) and deduce from the comparison of second equation (61) with similarity solution (47) that

$$
W_{3}\left(-1, \lambda_{3}\right)=0
$$


Thus, the unknown at the onset curvilinear interaction zone PQTR in the $(x t)$ plane maps to the prescribed rectangle $P Q T R$ in the hodograph $\left(\lambda_{1} \lambda_{3}\right)$ plane (Fig. 9, right) exactly as it happens in the problem of the interaction of two simple waves in classical gas dynamics (see e.g. [20]). We also note that, in contrast to the original free-boundary matching conditions (58), (59) for the Riemann invariants $\lambda_{j}(x, t)$, the boundary conditions for the functions $W_{1,3}\left(\lambda_{1}, \lambda_{3}\right)$ are linear (i.e. they do not depend on the particular solution).

To deduce boundary conditions for the EPD equation (60) from conditions (63), (64) for the Tsarev equations (35) we use the relations (36) between $W_{1,3}\left(\lambda_{1}, \lambda_{3}\right)$ and $g\left(\lambda_{1}, \lambda_{3}\right)$. Then from (63) we obtain a simple ODE

$$
g\left(\lambda_{1}, A^{+}\right)-\frac{\mathfrak{L}\left(\lambda_{1}, 1, A^{+}, A^{+}\right)}{\partial_{1} \mathfrak{L}\left(\lambda_{1}, 1, A^{+}, A^{+}\right)} \partial_{1} g\left(\lambda_{1}, A^{+}\right)=l
$$

which is readily integrated to give the boundary value of the function $g\left(\lambda_{1}, \lambda_{3}\right)$ at $\lambda_{3}=A^{+}$:

$$
g\left(\lambda_{1}, A^{+}\right)=C_{1} \mathfrak{L}\left(\lambda_{1}, 1, A^{+}, A^{+}\right)+l=\frac{C_{1}}{\sqrt{A^{+}-\lambda_{1}}}+l,
$$

where $C_{1}$ is an arbitrary constant.

Next, from (64), (36) we find

$$
g\left(-1, \lambda_{3}\right)-\frac{\mathfrak{L}\left(-1,1, \lambda_{3}, A^{+}\right)}{\partial_{3} \mathfrak{L}\left(-1, \lambda_{3}\right)} \partial_{3} g\left(-1, \lambda_{3}\right)=0,
$$

so the solution is readily found as

$$
g\left(-1, \lambda_{3}\right)=C_{2} \mathfrak{L}\left(-1,1, \lambda_{3}, A^{+}\right)
$$

where $C_{2}$ is another arbitrary constant.

Conditions (66) and (68) represent the Goursat type characteristic boundary conditions for the EPD equation (60). Now, we have two arbitrary functions $\phi_{1,2}(\lambda)$ (see general solution (39)) and two arbitrary constants $C_{1,2}$ at our disposal to satisfy boundary conditions (63) and (64). We first observe that, according to Section 4.4., the function $g\left(-1, \lambda_{3}\right)$ has the meaning of the modulation phase shift in the incident DSW. Since this DSW is described by a centred simple wave modulation solution, this phase shift must be equal to zero. Thus we set $C_{2}=0$ so that condition (68) assumes the form

$$
g\left(-1, \lambda_{3}\right)=0
$$

in accordance with the phase shift requirement (46).

Now, the easiest way to proceed is to put $\phi_{2}(\lambda) \equiv 0$ and $a_{1}=-1$ in (39) so that the solution of the EPD equation (60) reduces to a single quadrature

$$
g=\int_{-1}^{\lambda_{1}} \frac{\phi_{1}(\lambda) d \lambda}{\sqrt{\left(\lambda_{3}-\lambda\right)\left(\lambda_{1}-\lambda\right)}} .
$$

Now we need to find $\phi_{1}(\lambda)$ and $C_{1}$ to satisfy two conditions (66) and (69).

Substitution of (70) into boundary condition (66) yields

$$
\int_{-1}^{\lambda_{1}} \frac{\phi_{1}(\lambda) d \lambda}{\sqrt{\left(A^{+}-\lambda\right)\left(\lambda_{1}-\lambda\right)}}=\frac{C_{1}}{\sqrt{A^{+}-\lambda_{1}}}+l,
$$


which is an Abel integral equation for $\phi_{1}(\lambda)$ (see e.g. [53]). We recall that

$$
\text { if } \int_{a}^{x} \frac{\phi(\xi)}{\sqrt{x-\xi}} d \xi=f(x), \quad \text { then } \quad \phi(x)=\frac{1}{\pi} \frac{d}{d x} \int_{a}^{x} \frac{f(\xi)}{\sqrt{x-\xi}} d \xi \text {. }
$$

Thus, the solution to (71) is readily obtained in the form

$$
\phi_{1}(\lambda)=\frac{1}{\pi \sqrt{\lambda+1}}\left(C_{1} \sqrt{\frac{A^{+}+1}{A^{+}-\lambda}}+l \sqrt{A^{+}-\lambda}\right) .
$$

Now one can see that condition (69) is satisfied by (70), (72) only if $\phi_{1}(-1)=0$, which implies that $C_{1}=-l \sqrt{A^{+}+1}$ and so finally

$$
\begin{aligned}
g\left(\lambda_{1}, \lambda_{3}\right) & =-\frac{l}{\pi} \int_{-1}^{\lambda_{1}} \frac{\sqrt{\lambda+1}}{\sqrt{\left(A^{+}-\lambda\right)\left(\lambda_{3}-\lambda\right)\left(\lambda_{1}-\lambda\right)}} d \lambda \\
= & \frac{2 l\left(A^{+}+1\right)}{\pi \sqrt{\left(A^{+}-\lambda_{1}\right)\left(\lambda_{3}+1\right)}}\left(\Pi_{1}(s, z)-\mathrm{K}(z)\right)
\end{aligned}
$$

where $\Pi_{1}(s, z)$ is the complete elliptic integral of the third kind (see, e.g. [53]) and

$$
z=\frac{\left(A^{+}-\lambda_{3}\right)\left(\lambda_{1}+1\right)}{\left(A^{+}-\lambda_{1}\right)\left(\lambda_{3}+1\right)}, \quad s=-\frac{\lambda_{1}+1}{A^{+}-\lambda_{1}} .
$$

Hence, the modulation solution describing the interaction of counter-propagating DSW and RW is given by the formulae

$$
\lambda_{2}=1, \quad \lambda_{4}=A^{+}, \quad x-V_{1,3}\left(\lambda_{1}, 1, \lambda_{3}, A^{+}\right) t=\left(1-\frac{\mathfrak{L}}{\partial_{1,3} \mathfrak{L}} \partial_{1,3}\right) g\left(\lambda_{1}, \lambda_{3}\right),
$$

where $g\left(\lambda_{1}, \lambda_{3}\right)$ is specified by $(73)$.

The interaction continues until the moment $t^{*}$ defined by the condition $x_{2}^{+}\left(t^{*}\right)=x_{1}^{-}\left(t^{*}\right)$ (the right edge of the RW coincides with the trailing edge of the DSW). It is clear from the Riemann invariant sketch in Fig. 8 that this will take place when one has $\lambda_{3}=1$ and $\lambda_{1}=A^{-}$simultaneously. Substituting $\lambda_{3}=1$ and $\lambda_{1}=A^{-}$into hodograph solution (75) we find after some algebra that

$$
\begin{gathered}
t^{*}=\frac{2 \sqrt{2} l \mathrm{E}(r)}{\pi\left(1-A^{-}\right) \sqrt{A^{+}-A^{-}}}, \quad \text { where } \quad r=\frac{\left(A^{+}-1\right)\left(A^{-}+1\right)}{2\left(A^{+}-A^{-}\right)}, \\
x^{*}=\left(1+\frac{A^{+}+A^{-}}{2}\right) t_{*}+p, \quad \text { where } \quad p=l\left(1-\frac{2 A^{+} \sqrt{A^{+}-A^{-}}}{\sqrt{A^{+}+1}\left(2 A^{+}-A^{-}-1\right)}\right) .
\end{gathered}
$$

\subsection{After interaction, $t>t^{*}$}

At $t=t^{*}$ the DSW exits the RW region and the two waves separate. 


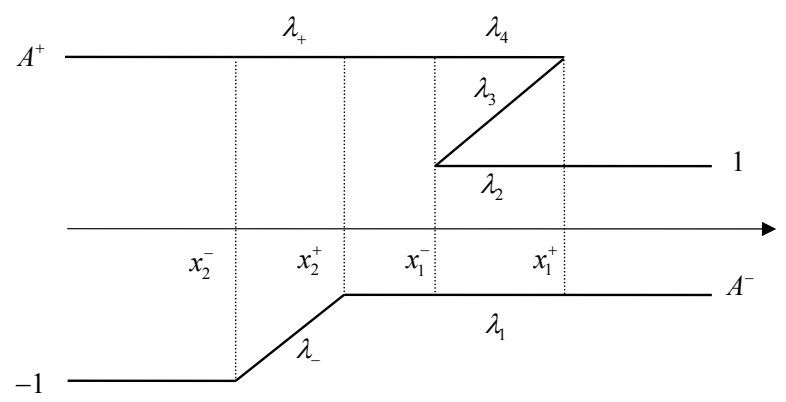

Figure 10: Schematic behaviour of the Riemann invariants after the interaction of the DSW and $\mathrm{RW}, t>t_{*}$.

\subsubsection{Refracted $D S W$}

The modulation solution describing the DSW after the separation is given by three constant invariants (see Fig. 10)

$$
\lambda_{1}=A^{-}, \quad \lambda_{2}=1, \quad \lambda_{4}=A^{+},
$$

while for the remaining one, $\lambda_{3}$, we have from (75) a simple-wave modulation solution (cf. (47))

$$
\begin{array}{r}
x=V_{3}\left(A^{-}, 1, \lambda_{3}, A^{+}\right) t+P\left(\lambda_{3}\right) \\
=\left(\frac{1}{2}\left(1+A^{-}+A^{+}+\lambda_{3}\right)+\frac{\left(A^{+}-\lambda_{3}\right)\left(\lambda_{3}-1\right)}{\left(\lambda_{3}-1\right)-\left(\lambda_{3}-A^{-}\right) \mu(m)}\right) t+P\left(\lambda_{3}\right),
\end{array}
$$

where

$$
m=\frac{\left(1-A^{-}\right)\left(A^{+}-\lambda_{3}\right)}{\left(A^{+}-1\right)\left(\lambda_{3}-A^{-}\right)}
$$

and the function $P(\xi)$ is found as

$$
\begin{array}{r}
P(\xi)=W_{3}\left(A^{-}, \xi\right)=\left(1-\frac{\mathfrak{L}\left(A^{-}, 1, \xi, A^{+}\right)}{\partial_{3} \mathfrak{L}\left(A^{-}, 1, \xi, A^{+}\right)} \partial_{3}\right) g\left(A^{-}, \xi\right) \\
=\frac{2 l}{\pi \sqrt{\left(A^{+}-A^{-}\right)(\xi+1)}}\left(\left(A^{+}+1\right) \Pi_{1}(p, z)+\frac{\left[\left(A^{+}\right)^{2}-1\right]\left(\xi-A^{-}\right) \mathrm{K}(z) \mu(y)-\left[\xi^{2}-1\right]\left[A^{+}-A^{-}\right] \mathrm{E}(z)}{\left(\xi-A^{-}\right)\left[(\xi-1)-\left(A^{+}-1\right) \mu(y)\right]}\right),
\end{array}
$$

where

$$
p=-\frac{A^{-}+1}{A^{+}-A^{-}}, \quad z=\frac{A^{-}+1}{A^{+}-A^{-}} \frac{A^{+}-\xi}{\xi+1}, \quad y=\frac{\left(1-A^{-}\right)\left(A^{+}-\xi\right)}{\left(A^{+}-1\right)\left(\xi-A^{-}\right)} .
$$

Expressions (82) are obtained from formulae (74), where one sets $\lambda_{1}=A^{-}, \lambda_{3}=\xi$, and the modulus $m$ in (81) is specified by (80) where $\lambda_{3}$ is replaced by $\xi$. Thus, as a result of the interaction, the DSW is no longer described by the similarity modulation solution in the form of an expanding centred fan but rather becomes a general simple wave solution of the modulation system corresponding to the following initial-value problem for the NLS equation (3):

$$
\lambda_{-}(x, 0)=A^{-}, \quad \lambda_{+}(x, 0)=P^{-1}(x),
$$


$P^{-1}(x)$ being inverse of the function $x=P\left(\lambda_{+}\right)$. The function $P\left(\lambda_{+}\right)$in (79) represents the DSW de-centring distribution acquired as a result of the interaction with the RW. It is directly related to the modulation phase shift distribution $\theta_{0}(x, t)$ via $(45)$, (46). It is not difficult to verify that $P(\xi) \equiv 0$ for $A^{-}=-1$. This is exactly what one should expect since when $A^{-}=-1$, there is no RW is generated and, therefore, there is no DSW refraction.

The boundaries $x_{1}^{-}$and $x_{1}^{+}$of the refracted DSW are found by setting in (79) $\lambda_{3}=1$ (i.e. $m=1$ ) and $\lambda_{3}=A^{+}$(i.e. $m=0$ ) respectively

$$
x_{1}^{-}=\left(1+\frac{A^{-}+A^{+}}{2}\right) t+P(1), \quad x_{1}^{+}=\left(2 A^{+}-\frac{\left(1-A^{-}\right)^{2}}{2\left(2 A^{+}-1-A^{-}\right)}\right) t+P\left(A^{+}\right) .
$$

The background density and the amplitude of the trailing dark soliton in the refracted DSW are (cf. (50))

$$
n_{s r}=\frac{1}{4}\left(A^{+}-A^{-}\right)^{2}, \quad a_{s r}=\left(A^{+}-1\right)\left(1-A^{-}\right) .
$$

The intensity $I_{r}$ of the refracted DSW is determined from (51) where we set $n_{1}=n_{s r}$ and $n_{2}=\frac{1}{4}\left(1-A^{-}\right)^{2}$ (the latter is defined by the initial conditions (11)). Thus

$$
I_{r}=\left(\frac{A^{+}-A^{-}}{1-A^{-}}\right)^{2}
$$

\subsubsection{Refracted $R W$}

The solution for the refracted RW is found from the hodograph modulation solution (75) by setting in it $\lambda_{4}=\lambda_{+}=A^{+}, \lambda_{3}=\lambda_{2}=1, \lambda_{1}=\lambda_{-}\left(\right.$see (56)) and using that $V_{1}\left(\lambda_{1}, \lambda_{3}, \lambda_{3}, \lambda_{4}\right)=V_{-}\left(\lambda_{1}, \lambda_{4}\right)$ (see 28). As a result we get

$$
\lambda_{+}=A^{+}, \quad x=V_{-}\left(\lambda_{-}, A^{+}\right) t+G\left(\lambda_{-}\right)=\frac{3 \lambda_{-}+A^{+}}{2} t+G\left(\lambda_{-}\right),
$$

where the function $G(\xi)$ has the form

$$
\begin{array}{r}
G(\xi)=W_{1}\left(\xi, A^{+}\right)=\left(1-\frac{\mathfrak{L}\left(\xi, 1,1, A^{+}\right)}{\partial_{1} \mathfrak{L}\left(\xi, 1,1, A^{+}\right)} \partial_{1}\right) g\left(\xi, A^{+}\right) \\
=\frac{l \sqrt{2}}{\pi \sqrt{A^{+}-\xi}}\left[\left(A^{+}+1\right)\left(\Pi_{1}(n, r)-\mathrm{K}(r)\right)+2 \mathrm{E}(r)\right],
\end{array}
$$

where

$$
r=\frac{\left(A^{+}-1\right)(\xi+1)}{2\left(A^{+}-\xi\right)}, \quad n=-\frac{\xi+1}{A^{+}-\xi} .
$$

Similar to the refracted DSW, the refracted RW is no longer described by a centred fan solution but rather by a general simple-wave solution of the shallow-water system (5), (6) with the 'effective' initial conditions $\lambda_{+}=1$ and $\lambda_{-}(x, 0)$ given by the function inverse to the refraction shift function $G\left(\lambda_{-}\right)$.

The boundaries of the refracted RW are given by the expressions

$$
x_{2}^{-}=\frac{A^{+}-3}{2} t+G(-1), \quad x_{2}^{+}=\frac{3 A^{-}+A^{+}}{2} t+G\left(A^{-}\right) .
$$




\subsubsection{Vacuum points}

As already was mentioned, an important property of the DSWs in the defocusing NLS flows is the possibility of the vacuum point(s) occurrence in the solutions for the problems not containing vacuum states in the initial data [34]. This effect has no analogue in both viscous SW dynamics and in the DSW dynamics in media with negative dispersion supporting bright solitons. Across the vacuum point, the flow speed changes its sign, which implies the generation of a counterflow. As a result, the DSW with a vacuum point inside it, unlike a regular DSW, no longer represents a single oscillatory wave of compression: the vacuum point separates the compression part propagating to the right and the oscillatory rarefaction wave propagating to the left [34]. The DSW counterflow due to the vacuum point occurrence has been recently observed in the experiments on nonlinear plane wave tunneling through a broad penetrable repulsive potential barrier (refractive index defect) in photorefractive crystals [56].

If we fix the state $n_{1}=1, u_{1}=0$ in front of the DSW (as we do for the incident wave), then, by increasing the density jump $n_{2}$ across the DSW we will be able to increase the DSW relative intensity only up to the value $I=4$ at which the vacuum point occurs at the DSW trailing edge [33]. If $n_{2}$ increases further, beyond the vacuum point threshold, the relative intensity of the compression part of the DSW decreases and, asymptotically as $n_{2} / n_{1} \rightarrow \infty$, vanishes so that the DSW completely transforms into the classical (smooth) left-propagating rarefaction wave [34]. This limit can alternatively be achieved by keeping the upstream state $n_{2}$ fixed and letting $n_{1} \rightarrow 0$ : then we arrive at the well-known solution of the classical shallow-water dam-break problem (see e.g. [22]).

Setting $A^{-}=-1$ we recover the already mentioned criterion $A^{+} \geq 3$ for the vacuum point occurrence in the incident DSW. If $a_{s r}=n_{s r}$, which by (85), yields the relation $A^{+}=2-A^{-}$, then the condition for the vacuum point appearance in the refracted DSW assumes the form

$$
A^{+} \geq 2-A^{-} \text {. }
$$

The regions of the $A^{-}, A^{+}$plane corresponding to different (with respect to the vacuum point appearance) flow configurations arising in the initial-value problem (3), (10) are presented in a diagram shown in Fig. 11. A particular flow evolution corresponding to Region II is shown in Fig. 12. We stress that, although the vacuum point appearance modifies the oscillatory DSW

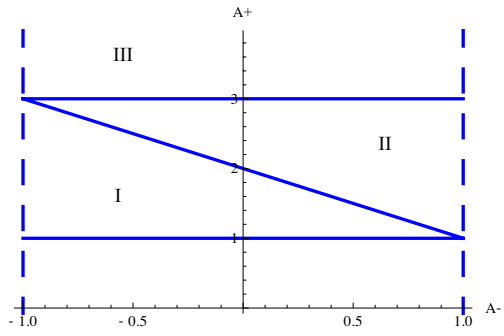

Figure 11: Regions in the plane of initial parameters $\left(A^{-}, A^{+}\right)$- the classification with respect to the vacuum point occurrence. (I): No vacuum points; (II): No vacuum points in the incident DSW, a vacuum point in the refracted DSW; (III): Vacuum points in both incident and refracted DSWs.

profile (the lower DSW density envelope becomes nonmonotonous and the velocity profile acquires a singularity at the vacuum point — see [34], [2]), all the dependencies of the DSW edge speeds, density jumps and trailing soliton amplitudes on the initial data $A^{+}, A^{-}$remain unchanged. 

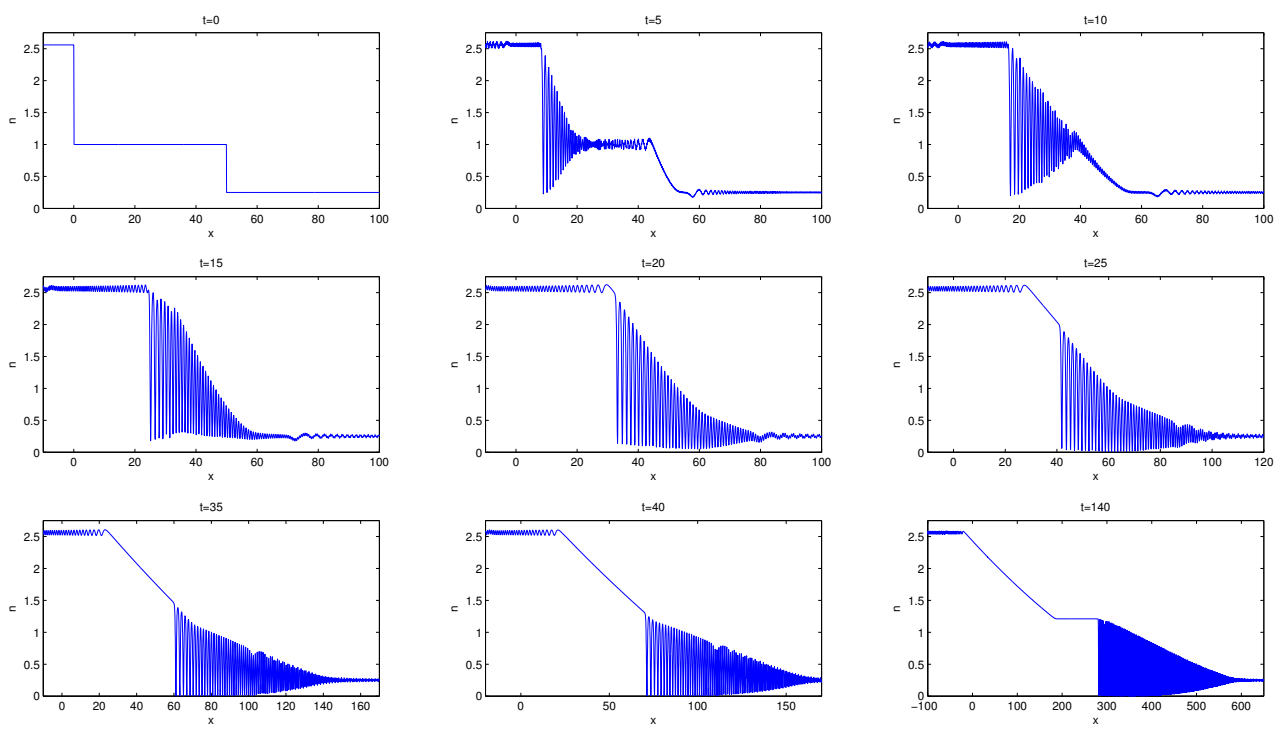

Figure 12: Evolution of the profile (10) with $A^{-}=0, A^{+}=2.2, l=50$ (Region II in Fig. 11) leading to the occurrence of a vacuum point in the refracted DSW.

\subsection{Key parameters of DSW refraction}

It is convenient to characterise the DSW refraction by three key parameters: the amplification coefficient $\nu$ which culd be defined as the ratio of the relative intensities (51) of the refracted and the incident DSWs, the acceleration coefficient $\sigma$ which we define as the difference between the values of the DSW trailing dark soliton speeds $s^{-}$after and before the interaction, and the refraction shift $d$ which is naturally defined as the phase shift of the DSW trailing soliton due to the DSW interaction with the RW (see Figs. 4,9).

For the first two parameters we readily have:

$$
\nu=\frac{I_{r}}{I_{0}}=\left(\frac{2\left(A^{+}-A^{-}\right)}{\left(1-A^{-}\right)\left(1+A^{+}\right)}\right)^{2}
$$

— see (86), (52), and

$$
\sigma=s_{r}-s_{0}=\left.\frac{d x_{1}^{-}}{d t}\right|_{t>t^{*}}-\left.\frac{d x_{1}^{-}}{d t}\right|_{t<t_{0}}=\frac{1+A^{-}}{2}>0
$$

- see (84), (49).

Here the subscripts ' 0 ' and ' $r$ ' refer to the incident and refracted waves respectively. Note that the determination of $\nu$ and $\sigma$ actually does not require knowledge of the full solution: both quantities are determined by the the transfer of the Riemann invariants through the DSW region. Interestingly, the acceleration coefficient $\sigma$ does not depend on the DSW strength before the interaction $\left(\sim A^{+}\right)$and is completely determined by the initial jump $A^{-}$of the Riemann invariant $\lambda_{-}$ across the RW. It also follows from (93) that, since $A^{-}>-1$, one has $\sigma>0$ i.e. the DSW is always accelerated as a result of the head-on collision with the RW (indeed, $\sigma>0$ implies acceleration of the trailing edge of the DSW and, therefore, acceleration of the DSW as a whole). The SW acceleration in the head-on collision with RW is also always the case in classical gas dynamics (see, e.g. [16]) as the SW meets the gas of decreasing density. 
Unlike the acceleration coefficient $\sigma$, the amplification coefficient $\nu$ can have both signs depending on the specific values of $A^{+}$and $A^{-}$chosen, the boundary between the regions of the DSW (relative) strengthening and attenuation being given by equation $A^{+}=\left(1-A^{-}\right) /\left(1+A^{-}\right)$. We also note that, while the amplification coefficient $\nu$ is formally defined for the full range of values of $A^{+}$and $A^{-}$, its original significance is retained only for the DSWs not containing vacuum points (see the discussion in the previous Section).

The function (see (81), (84))

$$
d\left(A^{+}, A^{-}\right)=P(1)=\frac{\sqrt{2} l}{\pi} \frac{\left(A^{+}+1\right)}{\sqrt{\left(A^{+}-A^{-}\right)}}\left(\Pi_{1}\left(p, z^{*}\right)-\mathrm{K}\left(z^{*}\right)\right),
$$

where (see $(82))$

$$
z^{*}=\frac{A^{-}+1}{A^{+}-A^{-}} \frac{A^{+}-1}{2}, \quad p=-\frac{A^{-}+1}{A^{+}-A^{-}},
$$

describes the refraction shift (see Fig. 4) of the trailing dark soliton in the DSW as a function of the initial parameters $A^{+}, A^{-}$. As a matter of fact, the determination of the refraction phase shift does require the knowledge of the full modulation solution in the interaction region. One can observe by comparing (94) with solution $g\left(\lambda_{1}, \lambda_{3}\right)(73)$, (74) of the EPD equation for the DSW-RW interaction region, that

$$
d\left(A^{+}, A^{-}\right)=g\left(A^{-}, 1\right),
$$

which corresponds to the value of $g$ at the moment $t=t^{*}$ (see (76)), when the DSW exits the interaction region - see Figs. 8, 9. This is, of course, expected from the general modulation phase shift consideration described in Section 4.4.

The dependencies of the refraction phase shift $d$ on $A^{-}$and $A^{+}$given by (94) along with direct numerical simulations data for the refraction shift are presented in Fig. 13. One can see that dependence of the refraction shift on the density jump across the RW (roughly proportional to the value of $A^{-}$) is much stronger than on the incident DSW strength (proportional to $A^{+}$). The plots and comparisons with numerics for $\sigma$ and $\nu$ will be presented in the next section as particular cases in the study of the DSW-RW interaction in the framework of a generalised, non-integrable version of the NLS equation.
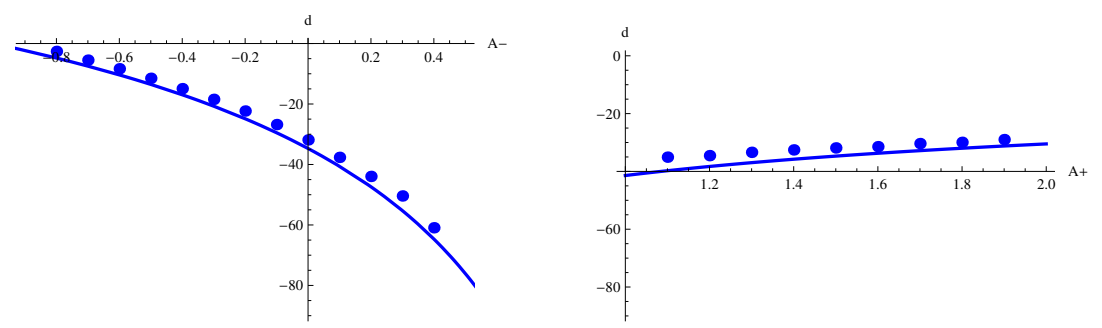

Figure 13: DSW refraction phase shift $d$. Left: dependence $d\left(A^{-}\right)$for fixed $A^{+}=1.5$, right: dependence $d\left(A^{+}\right)$for fixed $A^{-}=0$. Solid line: formula (94), circles: direct numerical simulations data.

One can trace certain analogy between the considered DSW-RW interaction and the two-soliton collisions in integrable systems: both interactions are elastic in the sense that they both can be interpreted in terms of the "exchange" of spectral parameters by the interacting waves so that the global spectrum in the associated linear scattering problem remains unchanged. In the DSW-RW interaction the role of isospectrality is played by the transfer of the constant values of appropriate Riemann invariants of the modulation system through the varying DSW and RW regions so that 
one can predict the jumps of density and velocity across the refracted DSW and RW without constructing the full modulation solution. At the same time, the DSW and RW do not simply pass through each other and "exchange" the constant Riemann invariants: there are additional phase shifts for both interacting waves, similar to the classical soliton phase-shifts.

\section{Refraction of dispersive shock waves in optical media with sat- urable nonlinearity}

\subsection{Formulation of the problem}

We now consider the NLS equation with saturable nonlinearity (hereafter called the sNLS equation)

$$
i \psi_{t}+\frac{1}{2} \psi_{x x}-\frac{|\psi|^{2}}{1+\gamma|\psi|^{2}} \psi=0
$$

where $\gamma>0$ is the saturation parameter. This equation describes, in a certain approximation, the one-dimensional propagation of a plane stationary light beam through a photo-refractive crystal (see e.g. [25], [26]). One should note that in the nonlinear optics context the role of the time variable $t$ is played by the spatial coordinate $z$ along the beam propagation direction while $x$ is the transversal coordinate. If the saturation effect is negligibly small $\left(\gamma|\psi|^{2} \ll 1\right)$, then the sNLS equation (97) reduces to the cubic NLS equation (1). The Madelung transformation (2) with $\epsilon=1$ maps equation (97) to the dispersive hydrodynamics system (cf. (3)),

$$
\begin{aligned}
n_{t}+(n u)_{x} & =0 \\
u_{t}+u u_{x}+\left(\frac{n}{1+\gamma n}\right)_{x}+\left(\frac{n_{x}^{2}}{8 n^{2}}-\frac{n_{x x}}{4 n}\right)_{x} & =0 .
\end{aligned}
$$

Here $n$ has the meaning of the light beam intensity and $u$ is the local value of the wave vector component transversal to the beam propagation direction. A detailed study of the periodic solutions to (98) can be found in [32]. In particular, the linear dispersion relation for the waves of infinitesimally small amplitude propagating against the constant background flow with $u=u_{0}, n=n_{0}$ has the form

$$
\omega=\omega_{0}\left(n_{0}, u_{0}, k\right)=k u_{0} \pm k \sqrt{\frac{n_{0}}{\left(1+\gamma n_{0}\right)^{2}}+\frac{k^{2}}{4}},
$$

where $\omega$ is the wave frequency and $k$ is the wavenumber.

In the dispersionless limit, system (98) can be cast in the diagonal form (5) with the Riemann invariants $\lambda_{ \pm}$and characteristic velocities $V_{ \pm}$expressed in terms of the hydrodynamic variables $n$ and $u$ as

$$
\lambda_{ \pm}=\frac{u}{2} \pm \frac{1}{\sqrt{\gamma}} \arctan \sqrt{\gamma n}, \quad V_{ \pm}=u \pm \frac{\sqrt{n}}{1+\gamma n} .
$$

When $\gamma \rightarrow 0$ expressions (100) go over to the shallow-water relationships (6), (7) (note the different normalization for the dispersionless Riemann invariants compared to that used in [32]).

Similar to (10), we specify the initial conditions for (98) in terms of two steps for the hydrodynamic Riemann invariants $\lambda_{ \pm}$

$$
\lambda_{+}(x, 0)=\left\{\begin{array}{ll}
A^{+} & \text {for } \quad x<0, \\
\frac{1}{\sqrt{\gamma}} \arctan \sqrt{\gamma} & \text { for } x>0 ;
\end{array} \quad \lambda_{-}(x, 0)= \begin{cases}-\frac{1}{\sqrt{\gamma}} \arctan \sqrt{\gamma} & \text { for } x<l, \\
A^{-} & \text {for } x>l,\end{cases}\right.
$$


where $A^{+}>\frac{1}{\sqrt{\gamma}} \arctan \sqrt{\gamma}$, and $-\frac{1}{\sqrt{\gamma}} \arctan \sqrt{\gamma}<A^{-}<\frac{1}{\sqrt{\gamma}} \arctan \sqrt{\gamma}$. The special values of $\lambda_{+}$ for $x>0$ and $\lambda_{-}$for $x<l$ are chosen such that initially the DSW and RW will propagate into an undisturbed "gas" (indeed one can readily see that $n=1, u=0$ in the middle region $0<x<l$ (cf. (11)).

Numerical simulations show that evolution (98), (101) for a broad range of initial data parameters $A^{ \pm}$leads to the same qualitative DSW refraction scenario as in the cubic NLS case studied in previous sections. The quantitative characteristics of the DSW refraction, however, now depend not only on the initial conditions but also on the saturation parameter $\gamma$ entering the sNLS equation. The results of [32] suggest that this dependence could be quite strong. Thus the DSW-RW interaction problem in the framework of the sNLS equation deserves a separate study. We also mention that knowledge of the effects of photorefractive saturation on the parameters of a DSW is especially important in the context of an all-optical modelling of BEC dynamics (see [3]).

Since the sNLS equation (98) is not integrable by the IST, the Riemann invariants are not available for the associated Whitham system and the modulation solution cannot be constructed by the methods used in previous Sections. An analytic description of the DSW refraction requires now a different technique. We shall take advantage of the theory of DSWs in photorefractive media developed in [32] and based on the 'dispersive shock fitting' method introduced in [31]. As already was mentioned, our specific interest here is to quantify the effect of the nonlinear saturation on the DSW refraction, and, in particular, on the parameters $\sigma$ and $\nu$ introduced above in the cubic NLS context (see (93), (92)).

\subsection{DSW transition relations}

The key ingredients of the dispersive shock fitting method of [31] in application to the sNLS equation (98) can be formulated as follows (see [32] for the details pertinent to the present study). Let the right-propagating DSW be confined to a finite region of space $x^{-}<x<x^{+}$and connect two constant hydrodynamic states $\left(n_{1}, u_{1}\right)$ at $x<x^{-}$and $\left(n_{2}, u_{2}\right)$ at $x>x^{+} ; n_{1}>n_{2}$. Such a DSW is called a simple DSW. At the trailing edge $x^{-}$the simple DSW assumes the form of a dark soliton moving with constant velocity $s^{-}$and at the leading edge $x^{+}$it degenerates into a vanishing amplitude linear wavepacket moving with constant group velocity $s^{+}, s^{+}>s^{-}$. The lines $x^{ \pm}=s^{ \pm} t$ represent free boundaries where the continuous matching of the mean flow $(\bar{n}, \bar{u})$ in the DSW region with the external constant states $\left(n_{1}, u_{1}\right)$ and $\left(n_{2}, u_{2}\right)$ occurs (in some cases it is more advantageous to formulate the matching conditions in terms of the mean density $\bar{n}$ and the mean momentum $\overline{n u}$ - see e.g. [2]).

The simple DSW transition between the hydrodynamic states $\left(n_{1}, u_{1}\right)$ and $\left(n_{2}, u_{2}\right)$ is described by the following relationships:

- The value of the Riemann invariant $\lambda_{-}$is conserved across the DSW ,

$$
\left.\lambda_{-}\right|_{x=x^{-}}=\left.\lambda_{-}\right|_{x=x^{+}},
$$

i.e.

$$
\frac{u_{1}}{2}-\frac{1}{\sqrt{\gamma}} \arctan \sqrt{\gamma n_{1}}=\frac{u_{2}}{2}-\frac{1}{\sqrt{\gamma}} \arctan \sqrt{\gamma n_{2}} \equiv \lambda_{-}^{0} .
$$

- The DSW edge speeds $s^{ \pm}$are defined by the kinematic conditions (cf. conditions (30) the cubic NLS case)

$$
s^{+}=\left.\frac{\partial \Omega}{\partial k}\right|_{\bar{n}=n_{2}, k=k^{+}} ; \quad s^{-}=\left.\frac{\widetilde{\Omega}}{\kappa}\right|_{\bar{n}=n_{1}, \kappa=\kappa^{-}} .
$$


The quantities $k^{+}$(the leading edge wavenumber) and $\kappa^{-}$(the trailing edge "soliton wavenumber" - the trailing soliton inverse half-width) in (104) represent the boundary values, $k^{+}=$ $k\left(n_{2}\right)$ and $\kappa^{-}=\kappa\left(n_{1}\right)$, of two functions $k(\bar{n})$ and $\kappa(\bar{n})$ satisfying the following ordinary differential equations (ODEs):

$$
\begin{array}{ll}
\frac{d k}{d \bar{n}}=\frac{\partial \Omega / \partial \bar{n}}{v_{+}(\bar{n})-\partial \Omega / \partial k}, & k\left(n_{1}\right)=0 ; \\
\frac{d \kappa}{d \bar{n}}=\frac{\partial \widetilde{\Omega} / \partial \bar{n}}{v_{+}(\bar{n})-\partial \widetilde{\Omega} / \partial \kappa}, & \kappa\left(n_{2}\right)=0 .
\end{array}
$$

Here

$$
\begin{gathered}
v_{+}(\bar{n})=V_{+}(\bar{n}, \bar{u}(\bar{n}))=\bar{u}(\bar{n})+\frac{\sqrt{\bar{n}}}{1+\gamma \bar{n}}, \\
\Omega(\bar{n}, k)=\omega_{0}(k, \bar{u}(\bar{n}), \bar{n})=k\left[\bar{u}(\bar{n})+\sqrt{\frac{\bar{n}}{(1+\gamma \bar{n})^{2}}+\frac{k^{2}}{4}}\right], \quad \widetilde{\Omega}(\bar{n}, \kappa)=-i \Omega(\bar{n}, i \kappa)
\end{gathered}
$$

and

$$
\bar{u}(\bar{n})=2\left(\lambda_{-}^{0}+\frac{1}{\sqrt{\gamma}} \arctan \sqrt{\gamma \bar{n}}\right) .
$$

- "Entropy" inequalities must hold ensuring that the hydrodynamic characteristics transfer data into the DSW region:

$$
V_{-}^{1}<s^{-}<V_{+}^{1}, \quad V_{+}^{2}<s^{+}, \quad s^{+}>s^{-} .
$$

Here $V_{ \pm}^{1} \equiv V_{ \pm}\left(n_{1}, u_{1}\right), V_{+}^{2} \equiv V_{+}\left(n_{2}, u_{2}\right)$ - see (100) for the definitions of $V_{ \pm}(n, u)$. We note that inequalities (110) represent the dispersive-hydrodynamic analogs of classical Lax's entropy conditions [51].

Relationships (102) - (110) enable one to 'fit' the DSW into the solution of the dispersionless limit equations without the knowledge of the detailed solution of the full dispersive system within the DSW region (much as in classical gas dynamics SW is fitted into the solution of the inviscid equations by means of the Rankine-Hugoniot conditions subject to Lax's entropy condition).

Using the speed-amplitude relationship for the photorefractive dark solitons obtained in [32] one can find the amplitude $a_{s}$ of the DSW trailing soliton. Setting the value $s^{-}(104)$ of the DSW trailing edge for the soliton velocity $c$ in formula (39) of [32] we obtain

$$
\left(s^{-}-u_{1}\right)^{2}=\frac{2\left(n_{1}-a_{s}\right)}{\gamma a_{s}}\left[\frac{1}{\gamma a_{s}} \ln \frac{1+\gamma n_{1}}{1+\gamma\left(n_{1}-a_{s}\right)}-\frac{1}{1+\gamma n_{1}}\right]
$$

(note: $u_{1}\left(n_{1}\right)$ is given by the simple DSW transition condition (103)).

\subsection{DSW refraction}

Our concern in this section will be with the calculations of two DSW refraction parameters: the DSW amplification and acceleration coefficients, defined earlier in (93) and (92) as

$$
\nu=I_{r} / I_{0} \quad \text { and } \quad \sigma=s_{r}^{-}-s_{0}^{-}
$$

respectively. We note that analytical determination of the refraction phase shift $d$ is, unfortunately, not feasible now as it requires knowledge of the full modulation solution, which is not available for the sNLS equation due to its nonintegrability so we shall present only numerical results for $d$. 


\subsubsection{Before interaction, $t<t_{0}$}

The previous analysis of [32] suggests that the decay of two spaced initial discontinuities (101) for the hydrodynamic Riemann invariants $\lambda_{ \pm}$would result, similar to the cubic NLS case, in a combination of a right-propagating simple DSW centred at $x=0$ and a left-propagating simple RW centred at $x=l$. Indeed, the simple DSW transition condition (103) is satisfied by the initial step at $x=0$, which implies a single DSW resolution of this step (provided the "entropy conditions" (110) are satisfied - see [32] for the justification); similarly, the jump at $x=l$ with constant Riemann invariant $\lambda_{+}$across it asymptotically produces a single left-propagating RW (see Fig. 14). Indeed, our numerical simulations of the sNLS equation (98) for a range of the saturation parameter $\gamma$ values confirm this scenario producing the plots qualitatively equivalent to that presented in Fig. 3.

Now, following [32], we derive the key parameters of the simple photorefractive DSW in the form convenient for the further application to the refraction problem.

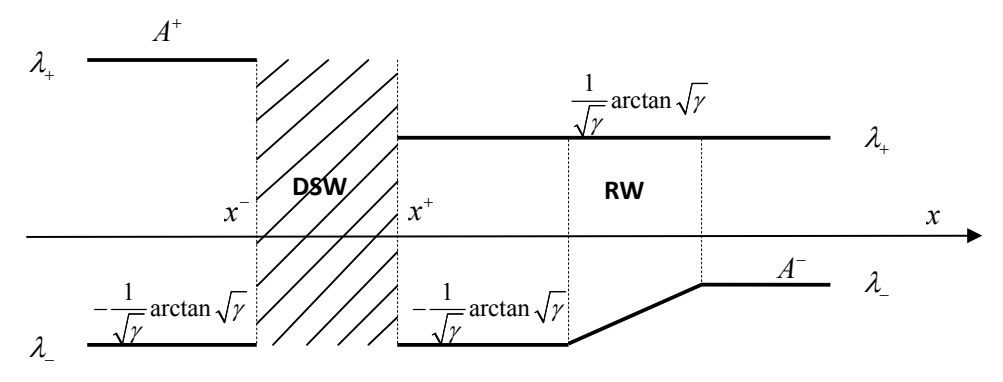

Figure 14: Distribution of the classical (dispersionless limit) Riemann invariants before the DSWRW interaction

To take advantage of formulae (104) - (109) for the speeds of the DSW edges we first need to find the constant states $\left(n_{1}, u_{1}\right)$ at $x<x^{-}$and $\left(n_{2}, u_{2}\right)$ at $x>x^{+}$defining the hydrodynamic jumps across the DSW. These are readily found from the the initial conditions (101) and the relationship (103) for the transfer of the Riemann invariant $\lambda_{-}$across the simple DSW. According to the initial conditions (101) the simple DSW must connect two hydrodynamic states with the same $\lambda_{-}=-\frac{1}{\sqrt{\gamma}} \arctan \sqrt{\gamma}$ while $\lambda_{+}=A^{+}$for $x<x^{-}$and $\lambda_{+}=\frac{1}{\sqrt{\gamma}} \arctan \sqrt{\gamma}$ for $x>x^{+}$(see Fig. 14). Then, using (103) and expressions (100) relating the Riemann invariants and the hydrodynamic variables $n, u$ we find

$$
n_{2}=1, \quad u_{2}=0, \quad n_{1}=\frac{1}{\gamma} \tan ^{2}\left(\frac{A^{+} \sqrt{\gamma}+\arctan \sqrt{\gamma}}{2}\right), \quad u_{1}=A^{+}-\frac{1}{\sqrt{\gamma}} \arctan \sqrt{\gamma}
$$

Thus, the $I_{0}$ of the incident DSW defined by (51) is simply

$$
I_{0}=\frac{1}{\gamma} \tan ^{2}\left(\frac{A^{+} \sqrt{\gamma}+\arctan \sqrt{\gamma}}{2}\right)
$$

Next, from (103) we have $\lambda_{-}^{0}=-\frac{1}{\sqrt{\gamma}} \arctan \sqrt{\gamma}$, which by (109) yields $\bar{u}(\bar{n})=\frac{2}{\sqrt{\gamma}}(\arctan \sqrt{\gamma \bar{n}}-$ $\arctan \sqrt{\gamma}$ ) and so completely defines, via (107), (108), ODEs (105), (106).

As was shown in [32], it is convenient to introduce a new variable $\widetilde{\alpha}$ instead of $\kappa$ using the substitution

$$
\widetilde{\alpha}=\sqrt{1-\frac{\kappa^{2}(1+\gamma \bar{n})^{2}}{4 \bar{n}}}
$$


which reduces ODE (106) to the form

$$
\frac{d \widetilde{\alpha}}{d \bar{n}}=-\frac{(1+\widetilde{\alpha})[1+3 \gamma \bar{n}+2 \widetilde{\alpha}(1-\gamma \bar{n})]}{2 \bar{n}(1+\gamma \bar{n})(1+2 \widetilde{\alpha})}, \quad \widetilde{\alpha}(1)=1 .
$$

The form (116) has an advantage of being a separable ODE when $\gamma=0$, which makes it especially useful for the asymptotic analysis for small $\gamma$. Once the function $\widetilde{\alpha}(\bar{n})$ is found, the velocity of the trailing soliton is determined by Eqs. (104), (108) as

$$
s_{0}^{-}=\frac{2}{\sqrt{\gamma}}\left(\arctan \sqrt{\gamma n_{1}}-\arctan \sqrt{\gamma}\right)+\frac{\sqrt{n_{1}}}{1+\gamma n_{1}} \widetilde{\alpha}\left(n_{1}\right),
$$

where $n_{1}$ is given by Eq. (113).

The amplitude of the trailing soliton is given by speed-amplitude relationship (111). Using (111), (117) and the relationship $u_{1}=\frac{2}{\sqrt{\gamma}}\left(\arctan \sqrt{\gamma n_{1}}-\arctan \sqrt{\gamma}\right)$ following from (103) one can derive the condition of the vacuum point occurrence at the DSW trailing edge (see [32]):

$$
\widetilde{\alpha}\left(n_{1}\right)=0
$$

Condition (118) yields, for a given value of the saturation parameter $\gamma$, the value of the initial density jump $n_{1}$ (and, therefore, of the parameter $A^{+}$- see (113)) corresponding to the vacuum point appearance at the DSW trailing edge. Say, for $\gamma=0.2$ this value of $A^{+}$is about 2.18 (cf. the critical value $A^{+}=3$ for $\gamma=0$ )

In conclusion of this Section we present an asymptotic expansion of $s_{0}^{-}$for small $\gamma$. First, to leading order we have from (116) a separable ODE

$$
\gamma=0: \quad \frac{d \widetilde{\alpha}}{d \bar{n}}=-\frac{1+\widetilde{\alpha}}{2 \bar{n}}, \quad \widetilde{\alpha}(1)=1,
$$

which is readily integrated to give

$$
\widetilde{\alpha}(\bar{n})=\frac{2}{\sqrt{\bar{n}}}-1 \equiv \widetilde{\alpha}_{0}(\bar{n})
$$

We now introduce

$$
\widetilde{\alpha}=\widetilde{\alpha}_{0}+\widetilde{\alpha}_{1}
$$

Substituting (121) into (116) and assuming $\widetilde{\alpha}_{1} \sim \gamma$ for $\gamma \ll 1$ we obtain to first order

$$
\frac{d \widetilde{\alpha}_{1}}{d \bar{n}}=-\frac{\widetilde{\alpha}_{1}}{2 \bar{n}}+\frac{4-3 \sqrt{\bar{n}}}{4-\sqrt{\bar{n}}} \frac{2 \gamma}{\sqrt{\bar{n}}} . \quad \widetilde{\alpha}_{1}(1)=0,
$$

Eq. (122) is readily integrated to give

$$
\widetilde{\alpha}_{1}(\bar{n})=\frac{2 \gamma}{\sqrt{\bar{n}}}\left(3(\bar{n}-1)+16(\sqrt{\bar{n}}-1)+64\left[\ln \frac{4-\sqrt{\bar{n}}}{3}\right]\right) .
$$

Now, substituting (121), (123) into (117) and using expansion of $n_{1}$ (113) for small $\gamma$ we obtain to first order

$$
s_{0}^{-}=\frac{A^{+}+1}{2}+\gamma\left(\frac{1}{12}\left[\left(A^{+}\right)^{3}+15\left(A^{+}\right)^{2}+219 A^{+}-245\right]+128 \ln \frac{7-A^{+}}{6}\right)+O\left(\gamma^{2}\right) .
$$

As one can see, expression (124) agrees to leading order with the cubic NLS result (49) for the trailing edge speed. We also notice that our perturbation approach formally breaks down for $A^{+} \geq 7$ because of the logarithmic divergence in Eq. (124) as $A^{+} \uparrow 7$ (we note that such values of $A^{+}$correspond to very large density jumps $\left(n_{1} / n_{2}>10\right)$ across the DSW - see [32]).

Formulae (113), (117) defining all the key parameters of the simple photorefractive DSW have been compared in [32] with direct numerical simulations data for a wide range of values of the saturation parameter $\gamma$ and a very good agreement was found. 


\subsubsection{After interaction, $t>t^{*}$}

Relations (102) - (110) describe a simple DSW transition between two constant states so they are not applicable to the varying transition in DSW-RW interaction zone. However, one should still be able to use these relations for the determination of the the key parameters of the refracted DSW when the interaction is over, provided no new waves is generated and the output pattern consists only of the pair of the refracted DSW and RW separated by a constant flow as it takes place in the Kerr nonlinearity case. In other words, relations (102) - (110) can be applied if the DSW-RW interaction is "clean" (elastic) on the level of the averaged Whitham description (which does not exclude the possibility of some constant-mean radiation due to non-integrability of the sNLS equation). If we accept this supposition (to be confirmed a-posteriori), then we can apply the transition relation (103) to the refracted DSW and determine the values of $n_{1}$ and $u_{1}$ in the 'plateau' region between the refracted DSW and RW. Since the refracted DSW propagates to the right into the region with $\lambda_{-}=A^{-}$(see the initial conditions (101) at $x \rightarrow+\infty$ ) one must have, by (102), the same $\lambda_{-}=A^{-}$across it, in the constant 'plateau' region.

Next, the refracted RW propagates to the left, into the region with $\lambda_{+}=A^{+}$(again, see initial conditions (101) at $x \rightarrow-\infty$ ) and, therefore $\lambda_{+}=A^{+}$everywhere through this wave and in the 'plateau' region. From the initial condition (103), the value of $\lambda_{-}$to the left of the RW is $\lambda_{-}=-\frac{1}{\sqrt{\gamma}} \arctan \sqrt{\gamma}$ and the value of $\lambda_{+}$to the right of the DSW is $\lambda_{+}=\frac{1}{\sqrt{\gamma}} \arctan \sqrt{\gamma}$. Thus, we arrive at the Riemann invariant diagram schematically shown in Fig. 15 (cf. diagram in Fig. 10 for the cubic NLS case).

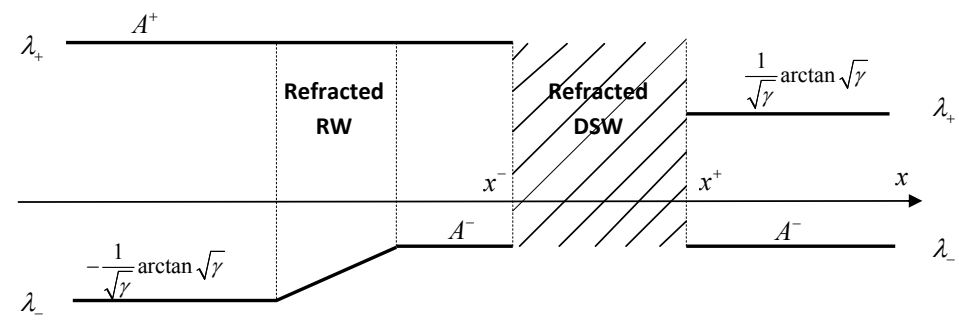

Figure 15: Distribution of the dispersionless limit Riemann invariants after the DSW-RW interaction.

Thus, using relationships (100) between the Riemann invariants $\lambda_{ \pm}$and the hydrodynamic variables $n, u$, one arrives at the set of equations determining the hydrodynamic states $\left(n_{1}, u_{1}\right)$ and $\left(n_{2}, u_{2}\right)$ at the trailing and leading DSW edges respectively:

$$
\begin{array}{r}
\frac{u_{1}}{2}+\frac{1}{\sqrt{\gamma}} \arctan \sqrt{\gamma n_{1}}=A^{+} ; \quad \frac{u_{1}}{2}-\frac{1}{\sqrt{\gamma}} \arctan \sqrt{\gamma n_{1}}=\frac{u_{2}}{2}-\frac{1}{\sqrt{\gamma}} \arctan \sqrt{\gamma n_{2}}=A^{-} ; \\
\frac{u_{2}}{2}+\frac{1}{\sqrt{\gamma}} \arctan \sqrt{\gamma n_{2}}=\frac{1}{\sqrt{\gamma}} \arctan \sqrt{\gamma} .
\end{array}
$$

So

$$
\begin{array}{r}
n_{1}=\frac{1}{\gamma} \tan ^{2}\left(\sqrt{\gamma} \frac{A^{+}-A^{-}}{2}\right), \quad u_{1}=A^{+}+A^{-}, \\
n_{2}=\frac{1}{\gamma} \tan ^{2}\left(\frac{1}{2} \arctan \sqrt{\gamma}-\frac{A^{-}}{2} \sqrt{\gamma}\right), \quad u_{2}=A^{-}+\frac{1}{\sqrt{\gamma}} \arctan \sqrt{\gamma} .
\end{array}
$$

To verify our key assumption about the "semi-classically clean" DSW-RW interaction in the sNLS equation case we have compared the values of the density and velocity in the region between 
the refracted DSW and RW obtained from direct numerical simulations of the sNLS equation with the predictions for $n_{1}$ and $u_{1}$ of formulae (126) based on this assumption. As one can see from Fig. 16 the the comparisons show an excellent agreement confirming our hypothesis for a range of values of $\gamma, A^{+}$and $A^{-}$. At the same, one can notice a small discrepancy visible at larger values of $A^{+}\left(A^{+} \gtrsim 1.7\right)$ in the plots for $\nu\left(A^{+}\right)$. This is connected with the occurrence of the vacuum point in the refracted DSW for sufficiently large density jumps across it. As was observed in [32], for large-amplitude photorefractive DSWs the Riemann invariant transition condition (103) is replaced by the classical Rankine-Hugoniot shock jump conditions so relation (126) holds only approximately for large $A^{+}$.
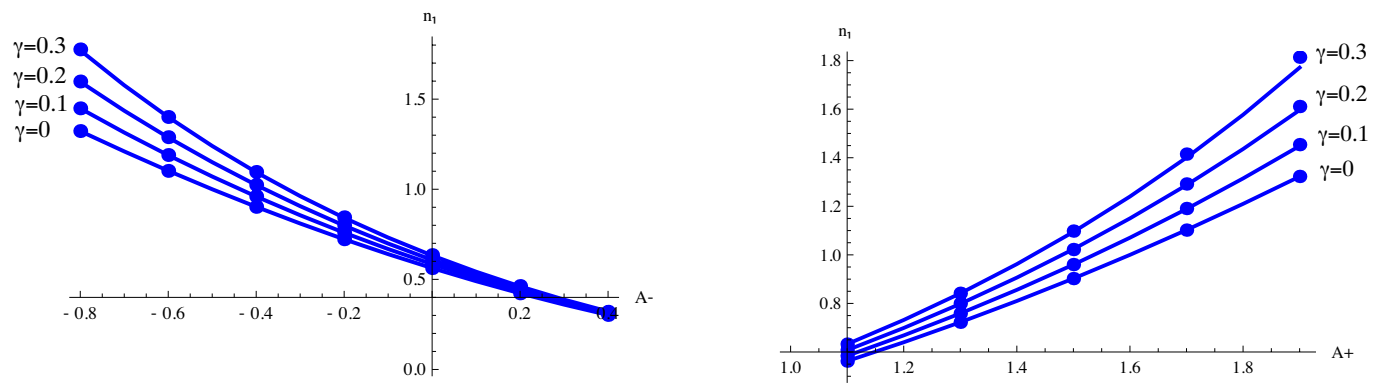

Figure 16: Density $n_{1}$ in the constant flow region between the refracted DSW and RW. Left: $n_{1}\left(A^{-}\right)$for fixed $A^{+}=1.5$; Right: $n_{1}\left(A^{+}\right)$for fixed $A^{-}=0$. Solid lines: analytic (modulation theory) curves; dots: direct numerical simulations data.

Now, we shall use general relationships (104) - (111) to derive the trailing soliton parameters in the refracted DSW.

Comparing (103) and (125) we find $\lambda_{-}^{0}=A^{-}$so expression (109) for $\bar{u}(\bar{n})$ assumes the form

$$
\bar{u}(\bar{n})=2\left(A^{-}+\frac{1}{\sqrt{\gamma}} \arctan \sqrt{\gamma \bar{n}}\right) .
$$

Substituting (127) into (107) and (108) and using the same change of variable (115) in ODE (106) we arrive at the same ODE (116) for the function $\widetilde{\alpha}(\bar{n})$ but now with a general boundary condition $\widetilde{\alpha}\left(n_{2}\right)=1$ since $n_{2} \neq 1$ for the refracted wave (see (126)). As before, this condition follows from the boundary condition for $\kappa$ in (106) and the relationship (115) between $\widetilde{\alpha}$ and $\kappa$. The velocity of the trailing soliton in the refracted DSW is determined by Eqs. (104), (108) as

$$
s_{r}^{-}=2\left(A^{-}+\frac{1}{\sqrt{\gamma}} \arctan \sqrt{\gamma n_{1}}\right)+\frac{\sqrt{n_{1}}}{1+\gamma n_{1}} \widetilde{\alpha}\left(n_{1}\right),
$$

where $n_{1}$ is now given by Eq. (126). Comparison for the dependence $s_{r}^{-}\left(A^{+}\right)$for a fixed value of $A^{-}=-0.8$ is presented in Fig. 18. One can see that the value of $s_{r}^{-}$quite strongly depends on the saturation parameter $\gamma$. Expanding $s_{r}^{-}$for small $\gamma$ we get (cf. (124))

$$
s_{r}^{-}=1+\frac{A^{-}+A^{+}}{2}+\gamma\left[\frac{2}{3} \Delta^{3}+4 \Delta^{2} \delta+32 \Delta \delta^{2}-\frac{112}{3} \delta^{3}+128 \delta^{3} \ln \frac{4-\Delta / \delta}{3}-\frac{1}{3}\right]+O\left(\gamma^{2}\right)
$$

Here $\Delta=\frac{A^{+}-A^{-}}{2}, \delta=\frac{1-A^{-}}{2}$. Again, one can see that the leading order of expansion (129) agrees with the cubic NLS result (84) as expected.

Given the value of $s_{r}^{-}$, the trailing dark soliton amplitude $a_{s}$ in the refracted DSW is found from formula (111). Comparisons of the analytically found values of $a_{s}$ for $\gamma=0.2$ with direct 


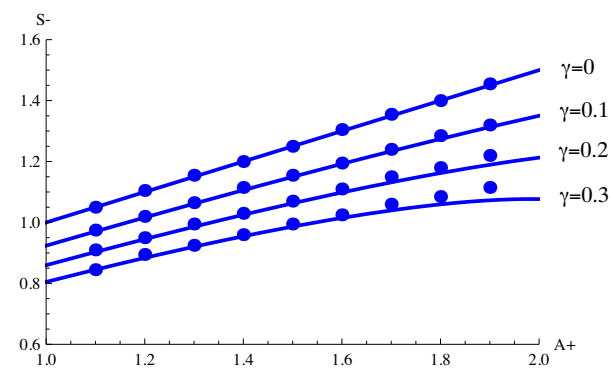

Figure 17: The refracted DSW trailing edge speed $s_{r}^{-}$as a function of an input parameter $A^{+}$for fixed $A^{-}=-0.8$. Solid lines: modulation solution (128); dots: numerical simulations data.

sNLS numerical simulation data are presented in Fig. 18. and show excellent agreement. Also, the dashed lines show the dependencies $a_{s}\left(A^{-}\right)$and $a_{s}\left(A^{+}\right)$for $\gamma=0$. As one can see, the nonlinearity saturation has strong effect on the refracted DSW soliton amplitude.
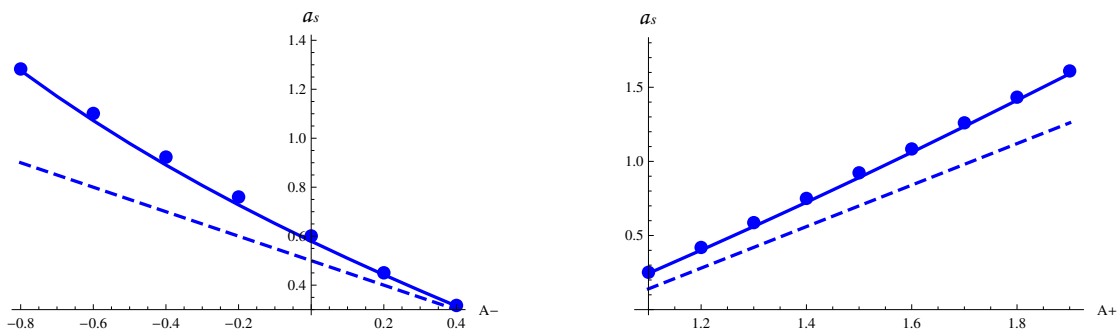

Figure 18: Trailing soliton amplitude $a_{s}$. Left: $a_{s}\left(A^{-}\right)$for $A^{+}=1.5$; Right: $a_{s}\left(A^{+}\right)$for $A^{-}=-0.4$. Solid line: analytic curve for $\gamma=0.2$; Dots: direct numerical simulations data for $\gamma=0.2$. Dashed line: the curve for $\gamma=0$.

The condition $a_{s}=n_{1}$ defining the vacuum point occurrence at the trailing edge of the refracted DSW, leads to the same equation (118), which was obtained earlier for the incident DSW, with the only (essential) difference that $n_{1}$ is now given by (126). The vacuum point regions diagram for $\gamma=0.2$ is presented in Fig. 19 .

Comparison with the analogous diagram for the Kerr nonlinearity case $\gamma=0$ (Fig. 11) shows that variations of the saturation parameter $\gamma$ have rather significant effect on the vacuum point appearance. Our numerical simulations confirm this conclusion. As already was mentioned, in the developed modulation theory we assume a semiclassically "clean" DSW-RW interaction, which, strictly speaking, applies only to the region I in Fig. 19. However, our comparisons show that, if the initial parameter $A^{+}$is not too large, the DSW fitting approach [31] based on the Riemann invariant transition condition (103) gives reasonably good quantitative predictions for the refracted DSW parameters in regions II and III as well.

\subsection{DSW refraction parameters}

The DSW amplification coefficient is defined as $\nu=I_{r} / I_{0}$, where the incident DSW relative intensity $I_{0}$ is given by (114). Using (126) the relative intensity of the refracted DSW is readily found in 


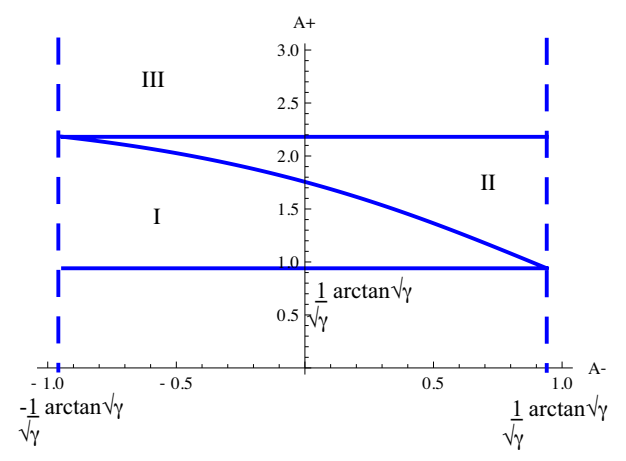

Figure 19: Regions of the plane of initial parameters $A^{-}, A^{+}$for $\gamma=0.2$ : (I) No vacuum points; (II) No vacuum points in the incident DSW, a vacuum point in the refracted DSW; (III) Vacuum points in both incident and refracted DSWs.

terms of the input parameters $A^{+}$and $A^{-}$as (see (51))

$$
I_{r}=\frac{n_{1}}{n_{2}}=\frac{\tan ^{2}\left(\sqrt{\gamma} \frac{A^{+}-A^{-}}{2}\right)}{\tan ^{2}\left(\frac{1}{2} \arctan \sqrt{\gamma}-\frac{A^{-}}{2} \sqrt{\gamma}\right)} .
$$

In Fig. 20 we present the dependencies $\nu\left(A^{-}\right)$and $\nu\left(A^{+}\right)$. One can see that the amplification coefficient (unlike individual parameters of the incident and refracted DSWs - see e.g. Fig. 16 above and Figs. 18, 19 below) shows a very weak dependence on the saturation parameter $\gamma$ for rather broad intervals of $A^{+}$and $A^{-}$so that one can safely use simple expression (92) obtained for $\gamma=0$. The direct numerical simulations fully confirm this conclusion (we do not present numerical points on Fig. 20 to avoid cluttering the plot).
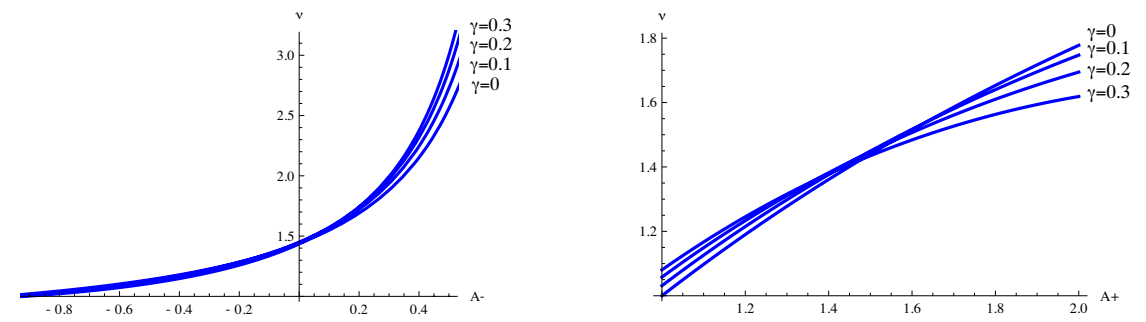

Figure 20: DSW amplification coefficient $\nu$. Left: $\nu\left(A^{-}\right)$at $A^{+}=1.5, A^{+}=1.5$. Right: $\nu\left(A^{+}\right)$at $A^{-}=0$;

Now we look at the behaviour of the acceleration coefficient $\sigma=s_{r}^{-}-s_{0}^{-}$, which is found analytically with the aid of formulae (128) and (117). The dependence $\sigma(\gamma)$ for $A^{+}=1.2, A^{-}=$ -0.7 (Region I in Fig. 20) is shown in Fig. 21. One can see that, similar to the amplification coefficient $\nu$, the dependence of $\sigma$ on $\gamma$ and $A^{+}$(i.e. on the intensity of the incident DSW) is quite weak. Indeed, the relative change of $\sigma$ does not exceed $10 \%$ over the broad interval of $\gamma$ from 0 to 0.5). Thus, at least in region I, one can safely assume the simple expression (93) $\sigma=\left(1+A^{-}\right) / 2$ obtained for the cubic nonlinearity case. The comparisons with numerics presented in Fig. 22 confirm this observation. To analytically quantify the deviations of the quite complicated general "photorefractive" dependence $\sigma\left(A^{+}, A^{-}, \gamma\right)$ from the simple dependence $\sigma=\left(1+A^{-}\right) / 2$ in the cubic nonlinearity case given by (93), we derive an asymptotic expansion for $\sigma$ for the case when 


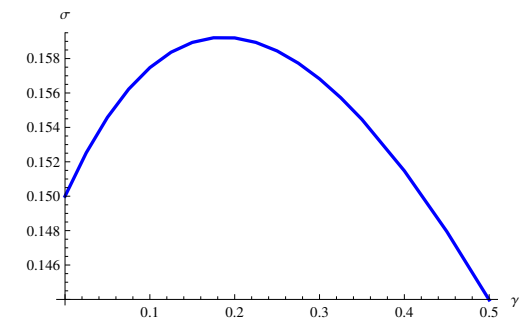

Figure 21: Analytical curve for the DSW acceleration coefficient $\sigma$ as a function of the saturation parameter $\gamma$ for $A^{+}=1.2, A^{-}=-0.7$.
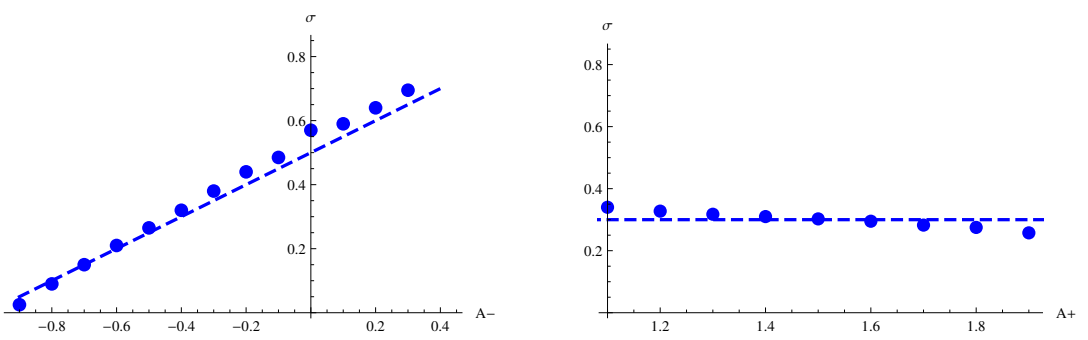

Figure 22: The DSW acceleration coefficient $\sigma$ as a function of input parameters $A^{-}$and $A^{+}$. Solid lines: analytic curves for $\gamma=0$; Cirles: numerical data for $\gamma=0.2$. Left: $\sigma\left(A^{-}\right)$at fixed $A^{+}=1.2$; Right: $\sigma\left(A^{+}\right)$for fixed $A^{-}=-0.4$

both interacting waves have small intensity. Introducing $\varepsilon_{+}$and $\varepsilon_{-}$by

$$
A^{-}=-\frac{1}{\sqrt{\gamma}} \arctan \sqrt{\gamma}+\varepsilon_{-}, \quad A^{+}=\frac{1}{\sqrt{\gamma}} \arctan \sqrt{\gamma}+\varepsilon_{+}
$$

and assuming $\varepsilon_{-} \ll 1, \varepsilon_{+} \ll 1$ we obtain from (124) and (129) on retaining second order terms,

$$
\sigma=s_{r}^{-}-s_{0}^{-}=\frac{\varepsilon_{-}}{2}+\varepsilon_{-} \gamma+O\left(\varepsilon_{-} \gamma^{2} ; \varepsilon_{-}^{2} \gamma ; \varepsilon_{-} \varepsilon_{+} \gamma\right) .
$$

One can see that expansion (132) does not contain terms proportional to $\varepsilon_{+} \gamma$, which implies that, for the interactions involving weak photorefractive DSW and RW, the acceleration $\sigma$ of the DSW up to second order does not depend on its initial intensity.
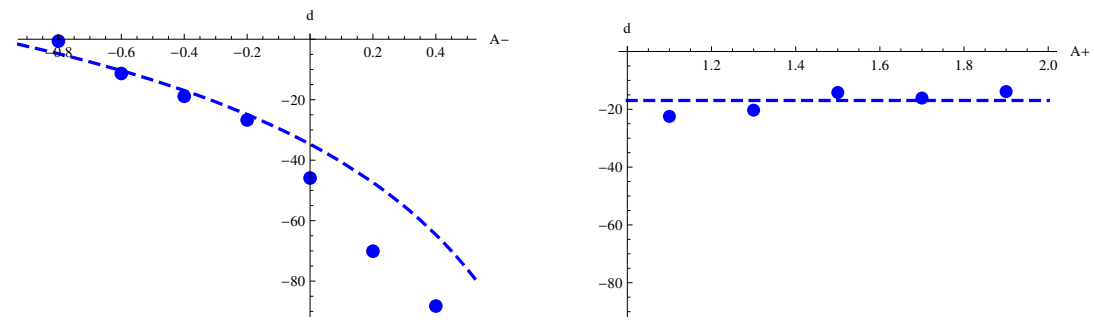

Figure 23: DSW refraction phase shift $d$. Left: dependence $d$ on $A^{-}$for fixed $A^{+}=1.5$; Right: dependence $d$ on $A^{+}$for fixed $A^{-}=0$. Dashed lines correspond to $\gamma=0$, cirles - to $\gamma=0.3$.

Finally, in Fig. 23 we present numerical values for the DSW refraction shift $d$ (see Fig. 4) taken for the particular value of $\gamma=0.3$. The numerics (circles) are put against the analytical curves 
$d\left(A^{-}, A^{+}\right)$defined by formula (94) for the cubic nonlinearity case, $\gamma=0$. One can see that, similar to other definitive DSW refraction parameters $\nu$ and $\sigma$, there is almost no dependence on $A^{+}$and $\gamma$ at a fixed value of $A^{-}$(roughly, the RW intensity), however, the departure of the dependence $d$ on $A^{-}$from the Kerr case $\gamma=0$ becomes more pronounced with growth of $A^{-}$.

\section{Conclusions}

In this paper, we have considered a dispersive counterpart of the classical gas dynamics problem of the interaction of a shock wave with a counter-propagating simple rarefaction wave often referred to as the shock wave refraction problem. Apart from the obvious contrast between the internal structures of viscous SWs and DSWs involved in the refraction process considered, there are some fundamental differences between the classical dissipative, and the present, dispersive conservative settings in terms of global quantitative descriptions of the SW/DSW refraction. The salient feature of the viscous SW refraction is the generation of the varying entropy wave resulting in a complicated system of the Rankine-Hugoniot shock conditions in the form of ordinary differential equations resolvable in most cases only by numerical means. Contrastingly, in conservative dispersive hydrodynamics, the thermodynamic entropy does not change and the jumps of the hydrodynamic quantities across the DSW are completely determined by the transfer of the Riemann invariants of the appropriate modulation Whitham equations along the characteristics, which makes possible a complete analytical asymptotic description of the flow.

Our study has been performed in the frameworks of the one-dimensional defocusing NLS equations with cubic nonlinearity (Eq. 1) and saturable nonlinearity (Eq. 97). To model a generic DSW-RW bidirectional interaction we have considered the initial-value problems for both NLS equations with initial data given by appropriate piecewise-constant distributions for the density (the wavefunction squared modulus) and the velocity (the wavefunction phase gradient). To single out the "pure" DSW-RW interaction we specified the initial data in the form of two steps for the "Eulerian" (dispersionless limit) Riemann invariants having the jumps of different polarity shifted with respect one another by a large distance $l$ (see Fig 2a).

For the integrable cubic nonlinearity case we have constructed exact modulation solutions, asymptotically $(t \gg 1)$ describing all stages of the bidirectional DSW-RW interaction in terms of the evolution of the Riemann invariants of the NLS-Whitham system. This was done by mapping the original nonlinear Gurevich-Pitaevskii type matching modulation problem to the Goursat problem for the classical linear Euler-Poisson-Darboux equation (60). Along with the modulation solution describing slow variations of the amplitude, the wavelength, the mean etc. in the DSW, we have derived explicit compact expressions for the DSW-RW refraction phase shifts, having certain analogy with the classical soliton phase-shifts in two-soliton collisions.

For the NLS equation with saturable nonlinearity, which is a typical model for the description of the light beam propagation through photorefractive optical materials, we have taken advantage of the DSW fitting method [31] applicable to non-integrable dispersive systems. This method was applied recently in [32] to the description of the simple-wave optical photorefractive DSWs and in the present study we extended it to the DSW-RW interaction. Our consideration of "nonintegrable" DSW refraction in the framework of the NLS equation with saturable nonlinearity (97) is based on the assumption (confirmed by direct numerical simulations) that the head-on DSW-RW interaction is "semiclassically elastic", i.e. is not accompanied by the generation of new DSWs or/and RWs. The comparisons of the key photorefractive DSW refraction parameters: the amplification coefficient $\nu$ and the acceleration coefficient $\sigma$ defined by formulae (112 a) and (112 b) respectively, with their Kerr $(\gamma=0)$ counterparts have revealed a rather weak dependence of these particular parameters on the saturation coefficient $\gamma$, which could prove useful for the experimental all-optical modelling of the BEC DSW refraction using photorefractive materials. 
A very good agreement of the predictions of the of our asymptotic analytical results with the direct numerical simulations in the DSW refraction problem provides further striking confirmation of the robustness of the modulation theory in non-integrable dispersive wave problems, now in the more complicated setting involving DSW-RW interactions.

We conclude with the remark that the methods used in this paper can also be applied to the problem of the overtaking DSW-RW interaction in the NLS flows. This problem, considered in [14] in the framework of the KdV equation using numerical solutions of the KdV-Whitham system can be solved analytically (both for the KdV and defocusing cubic NLS equatons) using the mentioned mapping of the Gurevich-Pitaevskii problem for the Whitham equations to the corresponding Goursat problem for the Euler-Poisson-Darboux equation. Due to a different dispersion sign and the possibility of the vacuum point occurrence within the DSW one can expect a number of qualitative and quantitative differences of the NLS overtaking interaction compared to those in the KdV flows.

\section{Acknowledgments}

V.V.Kh. thanks the London Mathematical Society for partial support of his visit to Loughborough University during which this work was started.

\section{References}

[1] E.A. Cornell, Talk at the "Conference on Nonlinear Waves, Integrable Systems and their Applications", (Colorado Springs, June 2005); http://jilawww.colorado.edu/bec/papers.html.

[2] M.A. Hoefer, M.J. Ablowitz, I. Coddington, E.A. Cornell, P. Engels, and V. Schweikhard, Dispersive and classical shock waves in Bose-Einstein condensates and gas dynamics, Phys. Rev. A 74, 023623 (2006).

[3] W. Wan, S. Jia, and J.W. Fleischer, Dispersive superfluid-like shock waves in nonlinear optics, Nature Physics, 3, 46 (2007).

[4] N. Ghofraniha, C. Conti, G. Ruocco, and S. Trillo, Shocks in nonlocal media, Phys. Rev. Lett. 99, 043903 (2007).

[5] P. Engels and C. Atherton, Stationary and nonstationary fluid flow of a Bose-Einstein condensate through a penetrable barrier, Phys. Rev. Lett. 99, 160405 (2007).

[6] K. Sasaki, N. Suzuki and H. Saito, Benard-von Karman vortex street in a Bose-Einstein condensate, Phys. Rev. Lett. 104, 150404 (2010).

[7] A. Amo et. al., Hydrodynamic solitons in polariton superfluids, arXiv:1101.2530 (2011)

[8] M. Hoefer and M. Ablowitz, Dispersive shock waves. Scholarpedia, 4(11):5562 (2009).

[9] R. Courant and K.O. Friedrichs, Supersonic flow and shock waves (Interscience, New York, 1948).

[10] L.D. Landau and E.M. Lifshitz, Fluid Mechanics (Pergamon Press, Oxford, 1987).

[11] J.J. Chang,P. Engels, and M. Hoefer, Formation of dispersive shock waves by merging and splitting Bose-Einstein condensates, Phys. Rev. Lett. 101170404 (2008). 
[12] A.V. Gurevich and L.P. Pitaevskii, Nonstationary structure of a collisionless shock wave Sov. Phys. JETP, 38, 291 (1974).

[13] G. A. El and R. H. J. Grimshaw, Generation of undular bores in the shelves of slowly-varying solitary waves, Chaos, 12:1015-1026 (2002).

[14] M. J. Ablowitz, D. E. Baldwin, M. A. Hoefer, Soliton generation and multiple phases in dispersive shock and rarefaction wave interaction, Phys. Rev. E, 80, 016603 (2009).

[15] R. Courant and K. O. Friedrichs Interaction of shock and rarefaction waves in one-dimensional media, National Defense Research Committee, Applied Mathematics Panel Report 38.1R, PB32196, AMG-1, (1943).

[16] H.E. Moses, The head-on collision of a shock wave and a rarefaction wave in one dimension, Journ. Appl. Phys. 19, 383 (1948).

[17] V. Ya. Arsenin, and N.N. Yanenko, On the interactions of progressive waves with shock waves in an isothermal gas, Dokl. Akad. Nauk SSSR, 109, (1956), 713716

[18] Z. Hasimoto, Interaction of a simple expansion wave with a shock wave in two-dimensional flows of a gas, Phys. Soc. Jpn. 19 (1964) pp. 1074-1078

[19] J. Rosciszewski, Calculations of the motion of non-uniform shock waves, Journ. Fluid Mech., 8, 337 (1960).

[20] B.L.Rozhdestvensky and N.N.Yanenko, The systems of quasilinear equations and their applications to gas dynamics. (Nauka, Moscow, 1978) [in Russian].

[21] L.V. Ovsyannikov, Lectures on the Foundations of Gas Dynamics (Nauka, Moscow, 1981) [in Russian].

[22] G.B. Whitham, Linear and Nonlinear Waves (Wiley-Interscience, New York, 1974).

[23] Yu.S. Kivshar and G.P. Agraval, Optical solitons. From Fibers to Photonic Crystals, (Academic Press, Amsterdam, 2003).

[24] L.P. Pitaevskii and S. Stringari, Bose-Einstein Condensation, Cambridge University Press, (Cambridge, 2003).

[25] S. Gatz and J. Herrmann, Soliton propagation in materials with saturable nonlinearity, J. Opt. Soc. Am. B 8, 2296 (1991).

[26] D.N. Christodoulides and M.I. Carvalho, Bright, dark, and gray spatial soliton states in photorefractive media, J. Opt. Soc. Am. B, 12, 1628 (1995)

[27] V.R. Kudashev and S.E. Sharapov, Inheritance of KdV symmetries under Whitham averaging and hydrodynamic symmetries of the Whitham equations, Theor. Math. Phys. 87, 358363 (1991).

[28] A.V. Gurevich, A.L. Krylov, and G.A. El, Riemann wave breaking in dispersive hydrodynamics, JETP Lett. 54, 102107.

[29] A.V. Gurevich, A.L. Krylov and G.A. El, Evolution of a Riemann wave in dispersive hydrodynamics, Sov. Phys. JETP, 74, 957-962 (1992). 
[30] F.R. Tian, Oscillations of the zero dispersion limit of the Kortewegde Vries equation, Commun. Pure Appl. Math. 46, 10931129 (1993).

[31] G.A. El, Resolution of a shock in hyperbolic systems modified by weak dispersion, Chaos, 15, 037103 (2005).

[32] G.A. El, A. Gammal, E.G. Khamis, R.A. Kraenkel, and A.M. Kamchatnov, Theory of optical dispersive shock waves in photorefractive media, Phys. Rev. A 76, 053813 (2007).

[33] A.V. Gurevich and A.L. Krylov, Dissipationless shock waves in media with positive dispersion, Sov. Phys. JETP 65, 944 (1987).

[34] G.A. El, V.V. Geogjaev, A.V. Gurevich, and A.L. Krylov, Decay of an initial discontinuity in the defocusing NLS hydrodynamics, Physica D 87, 186 (1995).

[35] Y. Kodama, The Whitham equations for optical communications: mathematical theory of NRZ, SIAM J. Appl. Math., 59, 2162 (1999).

[36] G. Biondini and Y. Kodama, On the Whitham equations for the defocusing nonlinear Schrodinger equation with step initial data, J. Nonlinear Sci., 16, 435 (2006).

[37] M.A. Hoefer, M.J. Ablowitz, P.Engels, Dispersive piston problem, Phys. Rev. Lett. 100, 084504 (2008).

[38] G.A. El, A.M. Kamchatnov, V.V. Khodorovskii, E.S. Annibale, and A. Gammal, Twodimensional supersonic nonlinear Schrödinger flow past an extended obstacle, Phys. Rev. E 80, 046317 (2009).

[39] A.M. Kamchatnov, S.V. Korneev, Flow of a Bose-Einstein condensate in a quasi-onedimensional channel under the action of a piston, Journ. Exp. Theor. Phys. 110, 110 (2010).

[40] G.A. El and A.M. Kamchatnov, Spatial dispersive sock waves generated in supersonic flow of Bose-Einstein condensate past slender body, Phys. Lett. A 350, 192 (2006); erratum: Phys. Lett. A 352, 554 (2006).

[41] G.A. El and A.L. Krylov, General solution of the Cauchy problem for the defocusing NLS equation in the Whitham limit, Phys. Lett. A 203, 77 (1995).

[42] A.M. Kamchatnov, R.A. Kraenkel, and B.A. Umarov, Asymptotic soliton train solutions of the defocusing nonlinear Schrodinger equation, Phys. Rev. E 66, 036609 (2002).

[43] S.P. Tsarev, On Poisson brackets and one-dimensional systems of hydrodynamic type, Soviet Math. Dokl. 31, 488 (1985).

[44] A.M. Kamchatnov, A. Gammal, and R.A. Kraenkel, Dissipationless shock waves in BoseEinstein condensates with repulsive interaction between atoms, Phys. Rev. A 69, 063605 (2004).

[45] M.A. Hoefer, P. Engels, and J.J. Chang, Matter-Wave Interference in Bose-Einstein Condensates: a dispersive hydrodynamic perspective Physica D 238, 1311 (2009).

[46] S. Jin, C.D. Levermore, D.W. McLaughlin, The semiclassical limit of the defocusing NLS hierarchy, Comm. Pure Appl. Math., 52, 613 (1999).

[47] A.M. Kamchatnov, Nonlinear Periodic Waves and Their Modulations (World Scientific, Singapore, 2000). 
[48] M.G. Forest and J.E. Lee, Geometry and modulation theory for periodic nonlinear Schrödinger equation, in Oscillation Theory, Computation, and Methods of Compensated Compactness, Eds. C. Dafermos et al, IMA Volumes on Mathematics and its Applications 2, (Springer, N.Y., 1987).

[49] M.V. Pavlov, Nonlinear Schrödinger equation and the Bogolyubov-Whitham method of averaging, Theor. Math. Phys. 71, 351 (1987).

[50] F. Tricomi, F. Differential equations (Blackie and Sons, Boston, 1961).

[51] P.D. Lax, Hyperbolic systems of conservation laws II, Comm. Pure Appl. Math. 10, 537 (1957).

[52] M.A. Hoefer, M.J. Ablowitz, Interactions of dispersive shock waves, Physica D 236, 44 (2007).

[53] M. Abramowitz and I.A. Stegun, Handbook of Mathematical Functions with Formulas, Graphs, and Mathematical Tables (New York, Dover Publications, 1972).

[54] A.V. Gurevich, A.L. Krylov and N.G. Mazur, Sov. Phys. JETP, Quasisimple waves in Kortewegde Vries hydrodynamics, 68, 966 (1989).

[55] A.V. Gurevich, A.L. Krylov, N.G. Mazur and G.A. El, Evolution of a localized perturbation in Korteweg - de Vries hydrodynamics, Sov. Phys. Doklady, 37, 198 (1992).

[56] W. Wan, S. Muenzel and J. W. Fleischer, Wave tunneling and hysteresis in nonlinear junctions, Phys. Rev. Lett. 104073903 (2010).

[57] Y. Kodama, V. U. Pierce, and F.-R. Tian, On the Whitham equations for the defocusing complex modified KdV equation, SIAM J. Math. Anal., 41, 26-58 (2008). 